\title{
Flavour geometry and effective Yukawa couplings in the MSSM
}

John Ellis, ${ }^{a}$ Robert N. Hodgkinson, ${ }^{b, c, e}$ Jae Sik Lee ${ }^{d}$ and Apostolos Pilaftsis ${ }^{e}$

a Theory Division, CERN,

CH-1211 Geneva 23, Switzerland

${ }^{b}$ Theory Group, Institute of Particle and Nuclear Studies, KEK,

1-1 Oho Tsukuba-shi, Ibaraki-ken 305-0801, Japan

${ }^{c}$ Departamento de Física Teòrica and IFIC, Universitat de València-CSIC,

E-46100, Burjassot, València, Spain

${ }^{d}$ Physics Division, National Centre for Theoretical Sciences, 300, Hsinchu, Taiwan

${ }^{e}$ School of Physics and Astronomy, University of Manchester, Manchester M13 9PL, United Kingdom

E-mail: John.Ellis@cern.ch, robert.hodgkinson@uv.es,

jslee@phys.cts.nthu.edu.tw, apostolos.pilaftsis@manchester.ac.uk

ABSTRACT: We present a new geometric approach to the flavour decomposition of an arbitrary soft supersymmetry-breaking sector in the MSSM. Our approach is based on the geometry that results from the quark and lepton Yukawa couplings, and enables us to derive the necessary and sufficient conditions for a linearly-independent basis of matrices related to the completeness of the internal $[\mathrm{SU}(3) \otimes \mathrm{U}(1)]^{5}$ flavour space. In a second step, we calculate the effective Yukawa couplings that are enhanced at large values of $\tan \beta$ for general soft supersymmetry-breaking mass parameters. We highlight the contributions due to non-universal terms in the flavour decompositions of the sfermion mass matrices. We present numerical examples illustrating how such terms are induced by renormalizationgroup evolution starting from universal input boundary conditions, and demonstrate their importance for the flavour-violating effective Yukawa couplings of quarks.

KEYWORDS: Supersymmetric Standard Model, B-Physics

ArXiv EPrint: 0911.3611 


\section{Contents}

1 Introduction 1

2 The flavour geometry of the MSSM 3

2.1 The geometric approach to flavour 3

$\begin{array}{lll}2.2 & \text { Flavour geometry of renormalization group effects } & 6\end{array}$

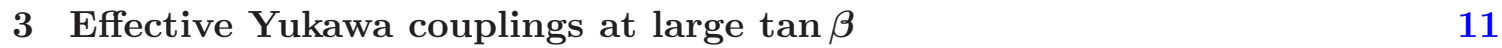

$\begin{array}{ll}3.1 & \text { Threshold corrections to Yukawa couplings } \\ \end{array}$

3.1.1 Down-type Quark Yukawa couplings 12

$\begin{array}{lll}3.1 .2 & \text { Up-type Quark Yukawa couplings } & 18\end{array}$

$\begin{array}{ll}3.1 .3 & \text { Lepton Yukawa couplings }\end{array}$

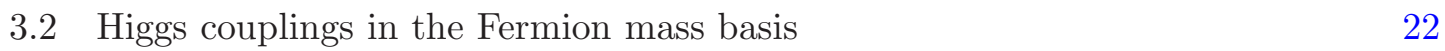

3.3 RG effects on FCNC Higgs-Boson couplings 25

$\begin{array}{llr}4 & \text { Conclusions } & 29\end{array}$

A Background Higgs-Field-Dependent mass matrices $\quad 30$

$\begin{array}{ll}\text { A.1 Squark mass-squared matrix } & 30\end{array}$

$\begin{array}{lll}\text { A.2 Chargino-Neutralino mass matrix } & 30\end{array}$

$\begin{array}{lll}\text { A.3 } & \text { Slepton mass-squared matrix } & 30\end{array}$

A.4 Higgs-boson mass-squared matrix 31

A.5 Quark mass matrix 31

\section{Introduction}

Supersymmetry (SUSY) is very attractive as a possible extension of the Standard Model, since it offers a mechanism for stabilizing the hierarchy between the gravitational and electroweak scale, would aid unification of the gauge couplings, provides a natural candidate for the astrophysical cold dark matter, predicts that the lightest Higgs boson should be relatively light, could explain the apparent discrepancy between the experimental measurement of $g_{\mu}-2$ and the prediction of the Standard Model, and is an essential ingredient of string theory [1-4]. However, the mechanism whereby SUSY could be broken is an open theoretical question and, in the absence of a convincing solution, the phenomenological description of SUSY breaking requires many unknown and apparently arbitrary parameters. These parameters are severely restricted by the conspicuous success of the Cabibbo-Kobayashi-Maskawa (CKM) description of flavour violation within the Standard Model $[5,6]$. Any model for new physics at the TeV scale, such as SUSY, must maintain 
this phenomenological success of the CKM mixing paradigm: this is the flavour problem of SUSY.

In this paper we discuss issues related to the SUSY flavour problem in the context of the minimal SUSY extension of the Standard Model, the MSSM, with general soft SUSYbreaking parameters. As is well known, these comprise the $\mathrm{U}(1)_{Y}, \mathrm{SU}(2)_{L}$ and $\mathrm{SU}(3)_{c}$ gaugino masses, $3 \times 3$ mass-squared matrices for the left-handed squarks and sleptons and for the right-handed squarks and charged sleptons, soft SUSY-breaking trilinear parameters corresponding to each of the Yukawa couplings of the Standard Model, soft SUSY-breaking masses for each of the two Higgs doublets $H_{u, d}$ and a soft bilinear SUSY-breaking parameter coupling the Higgs doublets.

The SUSY flavour problem concerns the structure of the mass-squared matrices for the squarks and sleptons. In general, loop diagrams involving sparticle exchanges will contribute to low-energy flavour-changing neutral-current (FCNC) interactions and CPviolating observables. Therefore, the agreement of flavour data with the predictions of the Standard Model, based on its Glashow-Iliopoulos-Maiani mechanism for suppressing FCNC interactions [7] and the Kobayashi-Maskawa mechanism for CP violation [6], imposes stringent conditions on the $3 \times 3$ mass-squared matrices for the squarks and sleptons and on the soft trilinear parameters. It is often assumed that all the SUSY flavour violation is due to the effects of the Yukawa coupling matrices, the minimal flavour violation (MFV) hypothesis [8-14]. One way to ensure that this hypothesis is obeyed is to require each of the mass-squared matrices and the trilinear parameters to be universal, i.e., proportional to the unit matrix in flavour space, $\mathbf{1}_{3}$, at some high input scale prior to their renormalization by the Yukawa couplings $\mathbf{h}_{u, d, e}$, which we denote here for brevity as $\mathbf{h}$. However, this is not the most general possibility, and in [15] the maximally CP-violating version of this MFV model (MCPMFV) has been introduced, which has 19 free parameters, including 6 CP-violating phases.

As pointed out in [15], generalizations that satisfy the MFV hypothesis may include non-universal terms in the mass-squared matrices $\widetilde{\mathbf{M}}_{Q, L, D, U, E}^{2}$ that are proportional to Hermitian products of Yukawa couplings $\mathbf{h h}$ and algebraic functions of them. The renormalization-group equations (RGEs) and their threshold corrections generate nonuniversal terms of this type [16-19], which are in the MFV spirit and may be consistent with the measurements of flavour-violating neutral interactions if their coefficients are not too large.

As we discuss in this paper, all deviations from universality in the mass-squared matrices $\widetilde{\mathbf{M}}_{Q, L, D, U, E}^{2}$ may be expressed in terms of the quadratic products $\mathbf{h h}$ and their quadratic and cubic combinations. They constitute complete, linearly-independent bases for the Hermitian matrices $\widetilde{\mathbf{M}}_{Q, L, D, U, E}^{2}$, and hence provide a convenient geometrical framework for discussing the SUSY flavour problem. For example, constraints on MFV scenarios within the MSSM may usefully be formulated as numerical bounds on the coefficients of expansions of the mass-squared matrices in these Hermitian bases. In a specific numerical example, we demonstrate how non-universal terms in these flavour decompositions are induced by renormalization-group evolution starting from universal input conditions at a Grand Unification scale. 
This formulation of SUSY flavour geometry can be used to simplify and systematize the calculations of a number of aspects of flavour violation in SUSY models. In this paper we discuss one specific such application, namely to effective quark Yukawa couplings at large $\tan \beta$ [20-27]. We present the complete sets of one-loop SUSY corrections to the selfenergies for the down- and up-quark and charged-lepton Yukawa couplings, highlighting the importance of the contributions of non-universal terms in the flavour decompositions of the squark and slepton mass matrices. We then use the same numerical example as previously to demonstrate the importance of these terms for effective Yukawa couplings in the MSSM.

The structure of this paper is as follows. In section 2 we demonstrate that the quadratic products of Yukawa couplings $\mathbf{h h}$ and their quadratic and cubic combinations constitute a basis in flavour space for soft SUSY-breaking contributions to the squark and slepton mass-squared matrices. Then, in section 3 we discuss the effective quark Yukawa couplings at large $\tan \beta$ and the numerical importance of flavour-non-diagonal terms in these masssquared matrices. Finally, the main results of our paper are summarised in section 4 .

\section{The flavour geometry of the MSSM}

In this section we discuss several new aspects of flavour geometry in the MSSM. First, we describe our geometric approach to the flavour decomposition of a general soft SUSYbreaking sector in terms of Yukawa couplings. We then illustrate within a specific MCPMFV scenario how this approach can be used to study quantitatively the flavour structure of renormalization-group (RG) effects on the soft SUSY-breaking matrices.

\subsection{The geometric approach to flavour}

To set the stage, we first introduce the superpotential of the MSSM:

$$
W_{\mathrm{MSSM}}=\widehat{U}^{C} \mathbf{h}_{u} \widehat{Q} \widehat{H}_{u}+\widehat{D}^{C} \mathbf{h}_{d} \widehat{H}_{d} \widehat{Q}+\widehat{E}^{C} \mathbf{h}_{e} \widehat{H}_{d} \widehat{L}+\mu \widehat{H}_{u} \widehat{H}_{d},
$$

where $\widehat{H}_{u, d}$ are the two Higgs chiral superfields, and $\widehat{Q}, \widehat{L}, \widehat{U}^{C}, \widehat{D}^{C}$ and $\widehat{E}^{C}$ are the leftand right-handed superfields related to up- and down-type quarks and charged leptons. The Yukawa couplings $\mathbf{h}_{u, d, e}$ are $3 \times 3$ complex matrices describing the charged-lepton and quark masses and their mixings. Finally, the $\mu$ parameter in (2.1) describes the mixing of the Higgs supermultiplets; it has to be of the electroweak order for a natural realization of the Higgs mechanism.

The required breaking of SUSY in nature is described by the effective soft SUSYbreaking Lagrangian

$$
\begin{aligned}
-\mathcal{L}_{\text {soft }}= & \frac{1}{2}\left(M_{1} \widetilde{B} \widetilde{B}+M_{2} \widetilde{W}^{i} \widetilde{W}^{i}+M_{3} \tilde{g}^{a} \tilde{g}^{a}+\text { H.c. }\right)+\widetilde{Q}^{\dagger} \widetilde{\mathbf{M}}_{Q}^{2} \widetilde{Q}+\widetilde{L}^{\dagger} \widetilde{\mathbf{M}}_{L}^{2} \widetilde{L} \\
& +\widetilde{U}^{\dagger} \widetilde{\mathbf{M}}_{U}^{2} \widetilde{U}+\widetilde{D}^{\dagger} \widetilde{\mathbf{M}}_{D}^{2} \widetilde{D}+\widetilde{E}^{\dagger} \widetilde{\mathbf{M}}_{E}^{2} \widetilde{E}+M_{H_{u}}^{2} H_{u}^{\dagger} H_{u}+M_{H_{d}}^{2} H_{d}^{\dagger} H_{d} \\
& +\left(B \mu H_{u} H_{d}+\text { H.c. }\right)+\left(\widetilde{U}^{\dagger} \mathbf{a}_{u} \widetilde{Q} H_{u}+\widetilde{D}^{\dagger} \mathbf{a}_{d} H_{d} \widetilde{Q}+\widetilde{E}^{\dagger} \mathbf{a}_{e} H_{d} \widetilde{L}+\text { H.c. }\right) .
\end{aligned}
$$

In the above, $M_{1,2,3}$ are the soft SUSY-breaking masses associated with the $\mathrm{U}(1)_{Y}, \mathrm{SU}(2)_{L}$ and $\mathrm{SU}(3)_{c}$ gauginos, respectively. In addition, $M_{H_{u, d}}^{2}$ and $B \mu$ are the soft masses related 
to the Higgs doublets $H_{u, d}$ and their bilinear mixing. Finally, $\widetilde{\mathbf{M}}_{Q, L, D, U, E}^{2}$ are the $3 \times 3 \mathrm{soft}$ mass-squared matrices of squarks and sleptons, and $\mathbf{a}_{u, d, e}$ are the corresponding $3 \times 3$ soft Yukawa coupling matrices related to quark and lepton masses. ${ }^{1}$ In addition to the $\mu$ term, the unconstrained CP-violating MSSM contains 109 mass parameters.

In order to study the flavour structure of the MSSM, we first notice that under the unitary flavour rotations of the quark and lepton superfields,

$$
\widehat{Q}^{\prime}=\mathbf{U}_{Q} \widehat{Q}, \quad \widehat{L}^{\prime}=\mathbf{U}_{L} \widehat{L}, \quad \widehat{U}^{\prime C}=\mathbf{U}_{U}^{*} \widehat{U}^{C}, \quad \widehat{D}^{\prime C}=\mathbf{U}_{D}^{*} \widehat{D}^{C}, \quad \widehat{E}^{\prime C}=\mathbf{U}_{E}^{*} \widehat{E}^{C},
$$

the complete MSSM Lagrangian of the theory remains invariant provided the model parameters are redefined as follows:

$$
\begin{aligned}
\mathbf{h}_{u, d} & \rightarrow \mathbf{U}_{U, D}^{\dagger} \mathbf{h}_{u, d} \mathbf{U}_{Q}, \quad \mathbf{h}_{e} \rightarrow \mathbf{U}_{E}^{\dagger} \mathbf{h}_{e} \mathbf{U}_{L}, \\
\widetilde{\mathbf{M}}_{Q, L, U, D, E}^{2} & \rightarrow \mathbf{U}_{Q, L, U, D, E}^{\dagger} \widetilde{\mathbf{M}}_{Q, L, U, D, E}^{2} \mathbf{U}_{Q, L, U, D, E} \\
\mathbf{a}_{u, d} & \rightarrow \mathbf{U}_{U, D}^{\dagger} \mathbf{a}_{u, d} \mathbf{U}_{Q}, \quad \mathbf{a}_{e} \rightarrow \mathbf{U}_{E}^{\dagger} \mathbf{a}_{e} \mathbf{U}_{L}
\end{aligned}
$$

The remaining mass scales, $\mu, M_{1,2,3}, M_{H_{u, d}}^{2}$ and $B \mu$, do not transform under the unitary flavour rotations (2.3). Thus, in the absence of the Yukawa couplings and soft SUSYbreaking parameters, the MSSM possesses the flavour symmetry $[\mathrm{SU}(3) \times \mathrm{U}(1)]^{5}[8]$.

Given the flavour transformations (2.4), one may wonder whether the soft SUSYbreaking mass parameters, $\widetilde{\mathbf{M}}_{Q, L, U, D, E}^{2}$ and $\mathbf{a}_{u, d, e}$, can be expressed in terms of the Yukawa couplings $\mathbf{h}_{u, d, e}$. This question was first discussed in [15] and subsequently studied in detail in $[13,14]$. However, our approach and results differ from the algebraic method presented in $[13,14]$, which makes explicit use of the Cayley-Hamilton identities. Instead, our method is entirely geometric and so enables us to derive the necessary and sufficient conditions on the completeness of the flavour space.

To start with, let us first consider the left-handed squark mass matrix $\widetilde{\mathbf{M}}_{Q}^{2}$. For a given renormalization scale $M_{X}, \widetilde{\mathbf{M}}_{Q}^{2}$ may be entirely determined by the decomposition:

$$
\widetilde{\mathbf{M}}_{Q}^{2}\left(M_{X}\right)=\sum_{I=0}^{8} \widetilde{m}_{Q}^{2, I}\left(M_{X}\right) \mathbf{H}_{I}^{Q}\left(M_{X}\right)
$$

where the $\mathbf{H}_{I}^{Q}$ are the following $3 \times 3$ Hermitian matrices constructed out of the Yukawa coupling matrices:

$$
\begin{aligned}
\left\{\mathbf{H}_{I}^{Q}\right\}= & \left\{\mathbf{1}_{3}, \mathbf{h}_{u}^{\dagger} \mathbf{h}_{u}, \mathbf{h}_{d}^{\dagger} \mathbf{h}_{d},\left(\mathbf{h}_{u}^{\dagger} \mathbf{h}_{u}\right)^{2},\left(\mathbf{h}_{d}^{\dagger} \mathbf{h}_{d}\right)^{2},\left[\mathbf{h}_{u}^{\dagger} \mathbf{h}_{u}, \mathbf{h}_{d}^{\dagger} \mathbf{h}_{d}\right]_{+}, i\left[\mathbf{h}_{u}^{\dagger} \mathbf{h}_{u}, \mathbf{h}_{d}^{\dagger} \mathbf{h}_{d}\right]_{-},\right. \\
& \left.\mathbf{h}_{u}^{\dagger} \mathbf{h}_{u} \mathbf{h}_{d}^{\dagger} \mathbf{h}_{d} \mathbf{h}_{u}^{\dagger} \mathbf{h}_{u}, \mathbf{h}_{d}^{\dagger} \mathbf{h}_{d} \mathbf{h}_{u}^{\dagger} \mathbf{h}_{u} \mathbf{h}_{d}^{\dagger} \mathbf{h}_{d}\right\} .
\end{aligned}
$$

In the above, the index $I$ labels all the 9 matrices $\mathbf{H}_{I}^{Q}$, i.e. $I=0,1,2, \ldots, 8$, and $[A, B]_{ \pm}=$ $\frac{1}{2}(A B \pm B A)$ for two matrices $A$ and $B$. The mass-squared parameters $\widetilde{m}_{Q}^{2, I}\left(M_{X}\right)$ are all real

\footnotetext{
${ }^{1}$ The soft Yukawa coupling matrices $\mathbf{a}_{u, d, e}$ may alternatively be defined by the relation: $\left(\mathbf{a}_{u, d, e}\right)_{i j}=$ $\left(\mathbf{h}_{u, d, e} \mathbf{A}_{u, d, e}\right)_{i j}$, where the matrix elements $\left(\mathbf{A}_{u, d, e}\right)_{i j}$ are typically of order $M_{\mathrm{SUSY}}$ in gravity-mediated SUSY-breaking models. In this paper we will use both definitions for the soft SUSY-breaking Yukawa couplings.
} 
and parametrize the 9 independent elements of the $3 \times 3$ Hermitian mass matrix $\widetilde{\mathbf{M}}_{Q}^{2}\left(M_{X}\right)$. Hence, the matrices $\mathbf{H}_{I}^{Q}$ may be regarded as a complete and linearly-independent set of basis vectors (or matrices) in this 9-dimensional space. The selection of the flavour basis is not unique, but the choice made in (2.6) is minimal in terms of the number of the Yukawacoupling matrices $\mathbf{h}_{u, d}$ involved, and is symmetric under the exchange of $\mathbf{h}_{u}$ with $\mathbf{h}_{d}$, with the exception $\mathbf{H}_{6}^{Q}$, which is anti-symmetric.

The flavour space spanned by $\mathbf{H}_{I}^{Q}$ may be assigned a metric defined by

$$
g_{I J}^{Q}=\operatorname{Tr}\left(\mathbf{H}_{I}^{Q} \mathbf{H}_{J}^{Q}\right) .
$$

Using the basis (2.6), we have checked that the determinant of the $9 \times 9$-dimensional matrix $g_{I J}^{Q}$ does not vanish, i.e. $\operatorname{det}\left(g_{I J}^{Q}\right) \neq 0$, provided the Jarlskog determinant [28] is not zero,

$$
\operatorname{det} \mathbf{H}_{6}^{Q}=\operatorname{det}\left(i\left[\mathbf{h}_{u}^{\dagger} \mathbf{h}_{u}, \mathbf{h}_{d}^{\dagger} \mathbf{h}_{d}\right]_{-}\right) \neq 0 .
$$

The latter is a necessary and sufficient condition for the metric $g_{I J}^{Q}$ to be non-degenerate, and hence for the basis matrices $\left\{\mathbf{H}_{I}^{Q}\right\}$ defined in (2.6) to form a linearly-independent set. This means that any arbitrary form of $\widetilde{\mathbf{M}}_{Q}^{2}$ can always be expressed in terms of the basis matrices $\mathbf{H}_{I}^{Q}$. Given the soft squark mass-squared matrix $\widetilde{\mathbf{M}}_{Q}^{2}$ and the basis vectors $\mathbf{H}_{I}^{Q}$, one can project out the parameters $\widetilde{m}_{Q}^{2, I}$ as follows:

$$
\widetilde{m}_{Q}^{2, I}=g^{Q, I J} \operatorname{Tr}\left(\mathbf{H}_{J}^{Q} \widetilde{\mathbf{M}}_{Q}^{2}\right),
$$

where summation over repeated indices is understood and $g^{Q, I J}$ is the inverse metric of $g_{I J}^{Q}$, obeying the property $g^{Q, I K} g_{K J}^{Q}=\delta^{I}{ }_{J}$.

Under an unitary flavour rotation (2.3) of the left-handed quark superfields, the basis matrices $\mathbf{H}_{I}^{Q}$ transform as follows:

$$
\mathbf{H}_{I}^{Q} \rightarrow \mathbf{H}_{I}^{\prime Q} \equiv \mathbf{U}_{Q}^{\dagger} \mathbf{H}_{I}^{Q} \mathbf{U}_{Q}=\left(L^{Q}\right)_{I}^{J} \mathbf{H}_{J}^{Q},
$$

where $\left(L^{Q}\right)_{I}^{J}$ is a $9 \times 9$ real matrix which can be evaluated from

$$
\left(L^{Q}\right)_{I}^{J}=g^{Q, J K} \operatorname{Tr}\left(\mathbf{U}_{Q}^{\dagger} \mathbf{H}_{I}^{Q} \mathbf{U}_{Q} \mathbf{H}_{K}^{Q}\right) .
$$

Using (2.10), it is not difficult to show that the $9 \times 9$ real transformation matrix $\left(L^{Q}\right)_{I}^{J}$ has the property:

$$
\left(L^{Q}\right)_{I}^{K}\left(L^{Q}\right)_{J}^{M} g_{K M}^{Q}=g_{I J}^{Q} .
$$

The choice of basis made in (2.6) is not orthonormal, since $g_{I J}^{Q} \neq \alpha \delta_{I J}$, where $\alpha$ is an overall normalization constant. Had we chosen the orthonormal basis spanned by the wellknown SU(3) Gell-Man matrices, $\mathbf{H}_{I}^{Q}=\lambda^{I}$ (for $\left.I=1,2, \ldots, 8\right)$ and $\mathbf{H}_{0}^{Q}=\sqrt{\frac{2}{3}} \mathbf{1}_{3}$, for which $g_{I J}^{Q}=2 \delta_{I J}$, the matrix $\left(L^{Q}\right)_{I}^{J}$ would have taken the form of a $9 \times 9$ real orthogonal matrix.

One can, by analogy, define basis matrices for the remaining matrices in the soft SUSY-breaking sector. Specifically, the $3 \times 3$ squared mass matrix $\widetilde{\mathbf{M}}_{U}^{2}$ pertaining to the right-handed up-type squarks may be decomposed in terms of the basis vectors

$$
\begin{aligned}
& \left\{\mathbf{H}_{I}^{U}\right\}=\left\{\mathbf{1}_{3}, \mathbf{h}_{u} \mathbf{h}_{u}^{\dagger}, \mathbf{h}_{u} \mathbf{h}_{d}^{\dagger} \mathbf{h}_{d} \mathbf{h}_{u}^{\dagger},\left(\mathbf{h}_{u} \mathbf{h}_{u}^{\dagger}\right)^{2}, \mathbf{h}_{u}\left(\mathbf{h}_{d}^{\dagger} \mathbf{h}_{d}\right)^{2} \mathbf{h}_{u}^{\dagger}, \mathbf{h}_{u}\left[\mathbf{h}_{u}^{\dagger} \mathbf{h}_{u}, \mathbf{h}_{d}^{\dagger} \mathbf{h}_{d}\right]_{+} \mathbf{h}_{u}^{\dagger},\right. \\
& \left.i \mathbf{h}_{u}\left[\mathbf{h}_{u}^{\dagger} \mathbf{h}_{u}, \mathbf{h}_{d}^{\dagger} \mathbf{h}_{d}\right]_{-} \mathbf{h}_{u}^{\dagger}, \mathbf{h}_{u} \mathbf{h}_{u}^{\dagger} \mathbf{h}_{u} \mathbf{h}_{d}^{\dagger} \mathbf{h}_{d} \mathbf{h}_{u}^{\dagger} \mathbf{h}_{u} \mathbf{h}_{u}^{\dagger}, \mathbf{h}_{u} \mathbf{h}_{d}^{\dagger} \mathbf{h}_{d} \mathbf{h}_{u}^{\dagger} \mathbf{h}_{u} \mathbf{h}_{d}^{\dagger} \mathbf{h}_{d} \mathbf{h}_{u}^{\dagger}\right\} .
\end{aligned}
$$


Again, we have checked that the corresponding metric $g_{I J}^{U}$ defined as in (2.7) is nondegenerate provided the condition (2.8) holds true and $\operatorname{det} \mathbf{h}_{u} \neq 0$. Likewise, an appropriate basis $\left\{\mathbf{H}_{I}^{D}\right\}$ for $\widetilde{\mathbf{M}}_{D}^{2}$ may be obtained by replacing the Yukawa coupling matrix $\mathbf{h}_{d}$ with $\mathbf{h}_{u}$ and vice versa in (2.13). This basis is non-degenerate if both (2.8) and $\operatorname{det} \mathbf{h}_{d} \neq 0$ are satisfied.

Finally, the soft-trilinear Yukawa matrices $\mathbf{a}_{u, d}$ may also be expanded as follows:

$$
\mathbf{a}_{u}=\sum_{I=0}^{8} a_{u}^{I} \mathbf{h}_{u} \mathbf{H}_{I}^{Q}, \quad \mathbf{a}_{d}=\sum_{I=0}^{8} a_{d}^{I} \mathbf{h}_{d} \mathbf{H}_{I}^{Q},
$$

where $a_{u, d}^{I}$ are complex parameters. Again, one has to assume here that the condition (2.8) and $\operatorname{det} \mathbf{h}_{u, d} \neq 0$ are satisfied, so that the sets $\left\{\mathbf{h}_{u, d} \mathbf{H}_{I}^{Q}\right\}$ form complete bases.

Unlike the scalar quark sector, the MSSM scalar lepton sector cannot be expanded in a complete set of basis matrices, since the only available Yukawa coupling matrix is $\mathbf{h}_{e}$. However, if there exist right-handed neutrinos that interact with left-handed lepton superfields $\widehat{L}$ via the Yukawa couplings $\mathbf{h}_{\nu}$, a complete basis can be formed for describing $\widetilde{\mathbf{M}}_{L, E}^{2}$ and $\mathbf{a}_{e}$. In this case, one needs to perform the obvious replacements $\mathbf{h}_{d} \rightarrow \mathbf{h}_{e}$ and $\mathbf{h}_{u} \rightarrow \mathbf{h}_{\nu}$ in the corresponding quark-basis matrices defined in (2.6) and (2.13).

An interesting flavour scenario for the MSSM is that termed in [15] the Maximal $\mathrm{CP}$ and Minimal Flavour Violation (MCPMFV) scenario. It contains the following set of flavour-singlet mass scales at some input scale $M_{X}$ that may be identical with $M_{\mathrm{GUT}}$ :

$$
M_{1,2,3}, \quad M_{H_{u, d}}^{2}, \quad \widetilde{\mathbf{M}}_{Q, L, U, D, E}^{2}=\widetilde{M}_{Q, L, U, D, E}^{2} \mathbf{1}_{3}, \quad \mathbf{A}_{u, d, e}=A_{u, d, e} \mathbf{1}_{3},
$$

with the obvious identifications: $\widetilde{m}_{Q, L, U, D, E}^{2,0}=\widetilde{M}_{Q, L, U, D, E}^{2}$ and $a_{u, d, e}^{0}=A_{u, d, e}$. At energy scales below $M_{X}$, RG effects modify the flavour structure of the soft SUSY-breaking mass and trilinear matrices. Specifically, these matrices get shifted as follows:

$$
\delta \widetilde{\mathbf{M}}_{Q, L, U, D, E}^{2}=\widetilde{\mathbf{M}}_{Q, L, U, D, E}^{2}-\widetilde{M}_{Q, L, U, D, E}^{2} \mathbf{1}_{3}, \quad \delta \mathbf{a}_{u, d, e}=\mathbf{a}_{u, d, e}-\mathbf{h}_{u, d, e} A_{u, d, e}
$$

where $\widetilde{M}_{Q, L, U, D, E}^{2}=\frac{1}{3} \operatorname{Tr}\left(\widetilde{\mathbf{M}}_{Q, L, U, D, E}^{2}\right)$ and $A_{u, d, e}=\frac{1}{3} \operatorname{Tr}\left(\mathbf{h}_{u, d, e}^{-1} \mathbf{a}_{u, d, e}\right)$.

In the next section we present numerical examples of RG-generated flavour structures in the squark mass matrices and trilinear couplings. We do not enter into numerical calculations of the coefficients in a flavour decomposition of the soft SUSY-breaking parameters in the lepton sector, as these would be dependent on the model for the neutrino sector. In section 3 we then use the MFV basis decomposition developed here and the flavour shifts given in (2.16) to obtain the complete flavour-covariant structure of the threshold corrections to the effective Yukawa couplings at leading order.

\subsection{Flavour geometry of renormalization group effects}

We now present numerical calculations of the flavour structure of the soft SUSY-breaking mass and trilinear matrices for a specific family of MCPMFV scenarios with

$$
\begin{aligned}
\left|M_{1,2,3}\right| & =250 \mathrm{GeV}, \\
M_{H_{u}}^{2} & =M_{H_{d}}^{2}=\widetilde{M}_{Q}^{2}=\widetilde{M}_{U}^{2}=\widetilde{M}_{D}^{2}=\widetilde{M}_{L}^{2}=\widetilde{M}_{E}^{2}=(100 \mathrm{GeV})^{2}, \\
\left|A_{u}\right| & =\left|A_{d}\right|=\left|A_{e}\right|=100 \mathrm{GeV}, \quad \Phi_{A}^{\mathrm{GUT}} \equiv \Phi_{A_{u}}=\Phi_{A_{u}}=\Phi_{A_{e}}=0^{\circ},
\end{aligned}
$$


at the GUT scale, varying the input value of $\tan \beta$ between 10 and 50 . When $\tan \beta=10$, this choice of parameters corresponds approximately to benchmark point B [29] and SPS point 1a [30]. We also allow for various common values of the CP-violating gaugino phases, denoted by $\Phi_{M}$.

As a first example, figure 1 gives some representative numerical results for this choice of parameters, showing how the different coefficients $\widetilde{m}_{Q}^{2, I}\left(M_{X}\right)$ in the basis expansion (2.5) of the left-handed squark mass-squared matrix $\widetilde{\mathbf{M}}_{Q}^{2}\left(M_{X}\right)$ vary as functions of $\tan \beta$ when evaluated at the SUSY-breaking scale, for various values of $\Phi_{M}$. Note that the dotted (red) and dash-dotted (magenta) lines, corresponding to $\Phi_{M}=90^{\circ}$ and $270^{\circ}$ respectively, overlap for all coefficients except $\widetilde{m}_{Q}^{2,6}$ and $\widetilde{m}_{Q}^{2,7}$; the solid (black) and dashed (blue) lines corresponding to $\Phi_{M}=0^{\circ}$ and $180^{\circ}$ overlap for $\widetilde{m}_{Q}^{2,6}$.

Specifically, the top left panel of figure 1 demonstrates how the leading flavour-singlet piece $\widetilde{m}_{Q}^{2,0}$ varies with $\tan \beta$ : we see that the variation is well below the $\%$ level. Turning to the other panels of figure 1 , which display the ratios of the other coefficients $\widetilde{m}_{Q}^{2, I}$ to $\widetilde{m}_{Q}^{2,0}$, we note first that the coefficients are all $\mathcal{O}(1)$ or smaller, showing that our flavourgeometric expansion is well behaved. In particular, there are no unphysical divergences for large $\tan \beta$. The largest flavour-changing contributions are generated along the $\widetilde{m}_{Q}^{2,1}$ and $\widetilde{m}_{Q}^{2,2}$ directions, corresponding to the basis matrices with the fewest powers of $\mathbf{h h}$. Such a hierarchical structure amongst the coefficients can be understood in terms of an approximate iterative solution to the RGEs. Finally, we observe that the magnitude of $\widetilde{m}_{Q}^{2, I}$ is maximised when $\Phi_{M}=180^{\circ}$ for $I=1,5$.

Some remarks regarding the computational procedure followed here are in order. In our approach, we fix the $3 \times 3$ Yukawa matrices at the scale $m_{t}^{\text {pole }}$ by applying the boundary conditions

$$
\mathbf{h}_{u}\left(m_{t}^{\text {pole }}\right)=\frac{\sqrt{2}}{v} \widehat{\mathbf{M}}_{u}\left(m_{t}^{\text {pole }}\right), \quad \mathbf{h}_{d, e}\left(m_{t}^{\text {pole }}\right)=\frac{\sqrt{2}}{v} \widehat{\mathbf{M}}_{d, e}\left(m_{t}^{\text {pole }}\right) \mathbf{V}_{d, e}^{\dagger}\left(m_{t}^{\text {pole }}\right)
$$

where $\mathbf{V}_{d}$ is the physical CKM matrix, $\mathbf{V}_{e}^{\dagger}$ is the Pontecorvo-Maki-Nakagawa-Sakata (PMNS) matrix [31, 32], and the $\widehat{\mathbf{M}}_{f}$ are the diagonal fermion mass matrices. These couplings run to the scale $M_{\text {SUSY }}$ according to the SM RGEs, where they match as

$$
\begin{aligned}
\mathbf{h}_{u}\left(M_{\mathrm{SUSY}}^{+}\right) & =\mathbf{h}_{u}\left(M_{\mathrm{SUSY}}^{-}\right) / \sin \beta\left(M_{\mathrm{SUSY}}\right), \\
\mathbf{h}_{d, e}\left(M_{\mathrm{SUSY}}^{+}\right) & =\mathbf{h}_{d, e}\left(M_{\mathrm{SUSY}}^{-}\right) / \cos \beta\left(M_{\mathrm{SUSY}}\right),
\end{aligned}
$$

before running from the soft SUSY-breaking scale $M_{\mathrm{SUSY}}$ to $M_{\mathrm{GUT}}$ according to the RGEs of the MSSM. However, threshold corrections due to sfermion-gaugino and sfermionHiggsino exchange, discussed in detail in the next section, can modify the above relations so that we have instead at the soft SUSY-breaking scale,

$$
\begin{aligned}
\mathbf{h}_{u}\left(M_{\mathrm{SUSY}}\right) & =\frac{\sqrt{2}}{v \sin \beta} \widehat{\mathbf{M}}_{u}\left(M_{\mathrm{SUSY}}\right) \mathbf{R}_{u}^{-1}\left(M_{\mathrm{SUSY}}\right) \\
\mathbf{h}_{d, e}\left(M_{\mathrm{SUSY}}\right) & =\frac{\sqrt{2}}{v \cos \beta} \widehat{\mathbf{M}}_{d, e}\left(M_{\mathrm{SUSY}}\right) \mathbf{V}_{d, e}^{\dagger}\left(M_{\mathrm{SUSY}}\right) \mathbf{R}_{d, e}^{-1}\left(M_{\mathrm{SUSY}}\right),
\end{aligned}
$$



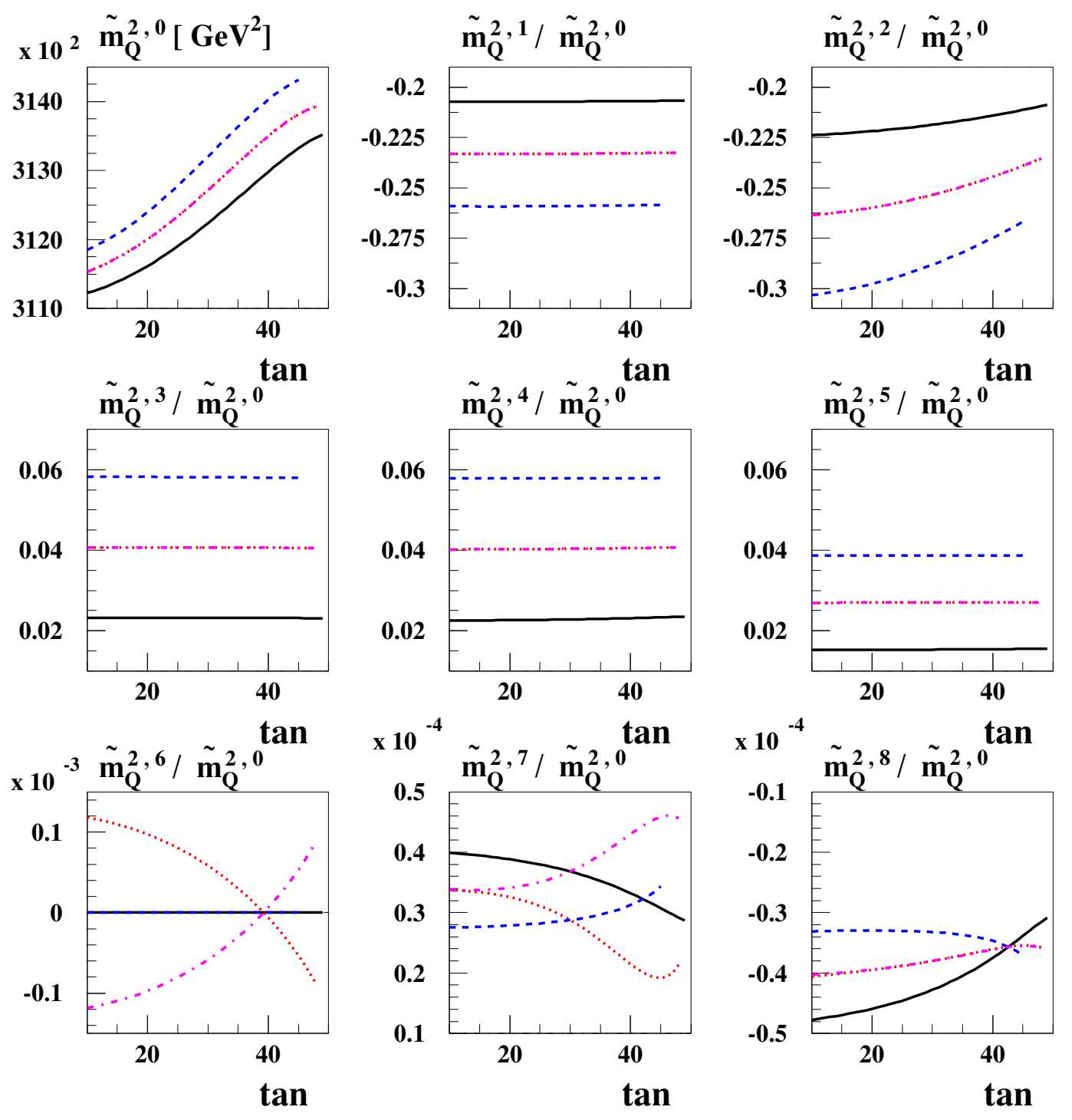

Figure 1. In the top left panel we display the variation of $\widetilde{m}_{Q}^{2,0}$ with $\tan \beta$, in the expansion (2.5) of the left-handed squark mass-squared matrix, as evaluated at the SUSY-breaking scale. In the other panels we display the variations with $\tan \beta$ of the ratios $\widetilde{m}_{Q}^{2, I} / \widetilde{m}_{Q}^{2,0}$. In each frame, the solid (black), dotted (red), dashed (blue), and dash-dotted (magenta) lines are for $\Phi_{M} \equiv \Phi_{1}=\Phi_{2}=\Phi_{3}=0^{\circ}$, $90^{\circ}, 180^{\circ}$, and $270^{\circ}$, respectively. The input MCPMFV SUSY-breaking parameters are taken as in $(2.17)$.

where the $\mathbf{R}_{u, d, e}$ matrices resum potentially large or $\tan \beta$-enhanced effects due to threshold corrections. Since these corrections depend upon the soft SUSY-breaking mass and trilinear matrices evaluated at the SUSY-breaking scale, rather than the GUT-scale, a full implementation of these effects would require an iterative solution to the RGEs. Instead, we follow here a more simplified approach and treat the threshold corrections as higher- 
order effects by neglecting their contribution to the RG running. Such an approximation is generally valid for most choices of the SUSY-breaking parameters (for exceptional regions of parameter space, see [33]).

One may think of certain extreme scenarios in which the threshold corrections lead to dramatic effects in the low-energy theory and some care is required in describing the flavour-geometry of such cases. For instance, one may consider a scenario in which the Yukawa matrices are real at the GUT scale. Such a scenario was previously considered in [34]. Then, Higgsino-mediated threshold effects will make the Yukawa couplings complex, and so a sizeable CP-violating phase for the CKM matrix can be generated, especially at large values of $\tan \beta$. In this situation, the condition (2.8) is not satisfied by the running Yukawa matrices above the SUSY-breaking scale, and these matrices cannot be used as a complete basis for projecting out the CP-violating flavour structure of the soft SUSYbreaking matrices. Instead, we may use the effective Yukawa couplings that include CPviolating threshold effects to decompose the soft SUSY-breaking mass matrices as normal. In such a scenario, the observed $\mathrm{CP}$ violation in the $K$ - and $B$-meson systems may be accounted for predominantly by the CP-violating soft SUSY-breaking sector.

Another extremal case would be to consider a scenario in which the effective Yukawa couplings at the top-quark mass scale are real, by arranging for $\mathbf{V}_{f}^{\dagger} \mathbf{R}_{f}^{-1}$ to be real for $f=d, e$. In this case, the flavour geometry of the soft SUSY-breaking matrices cannot be decomposed in our usual basis defined by the effective Yukawa couplings and we would be forced to choose a different basis, e.g. taking the threshold-uncorrected Yukawa matrices as defined above the SUSY scale. In such a scenario the CKM matrix is complex at the tree level and CP-violation may be mediated predominantly by the $W$ bosons according to the standard KM paradigm. In contrast, the $\tan ^{2} \beta$-enhanced Higgs-mediated effects would be purely CP-conserving.

Figure 2 gives some representative numerical results for the choice of parameters (2.17), showing how the RG-induced coefficients in the flavour decomposition of $\widetilde{\mathbf{M}}_{U}^{2}$ vary as functions of $\tan \beta$. We see that the lines corresponding to $\Phi_{M}=90^{\circ}$ and $270^{\circ}$ again overlap for the coefficients $I=0,3$, whilst those for $\Phi_{M}=0^{\circ}$ and $180^{\circ}$ are overlapping for $I=6,7$. We again observe a hierarchical structure amongst the flavour-changing components, although now with a bias towards the up-type quark Yukawa matrices. The coefficient $\widetilde{m}_{U}^{2,1}$, corresponding to the $\mathbf{h}_{\mathbf{u}} \mathbf{h}_{\mathbf{u}}^{\dagger}$ direction in flavour space, is here of order $\sim 50 \%$ of the leading flavour-singlet term. In contrast, the down-type Yukawa couplings first enter at the order $(\mathbf{h h})^{2}$ and are thus suppressed.

By analogy with figures 1 and 2, figures 3, 4 and 5 show for the same choice of parameters (2.17) the variations with $\tan \beta$ of the corresponding coefficients in the flavour decompositions of the right-handed down-squark mass-squared matrices, and the trilinear couplings $\mathbf{a}_{u, d}$, respectively. We see that the coefficients of $\widetilde{\mathbf{M}}_{D}^{2}$ exhibit a similar behaviour to those of $\widetilde{\mathbf{M}}_{U}^{2}$, favouring now the directions in flavour space corresponding to the downtype quark Yukawa matrices and consequently with a stronger dependence on $\tan \beta$. Note also the relative change in sign between $\widetilde{m}_{U}^{2,5}$ and $\widetilde{m}_{D}^{2,5}$. Turning to the trilinear couplings, we see a similar hierarchical pattern amongst the leading terms, with the largest non-singlet coefficient of $\mathbf{a}_{\mathbf{u}}\left(\mathbf{a}_{\mathbf{d}}\right)$ being $I=1(2)$, corresponding to the $\mathbf{h}_{\mathbf{u}}^{\dagger} \mathbf{h}_{\mathbf{u}}\left(\mathbf{h}_{\mathbf{d}}^{\dagger} \mathbf{h}_{\mathbf{d}}\right)$ direction in our 

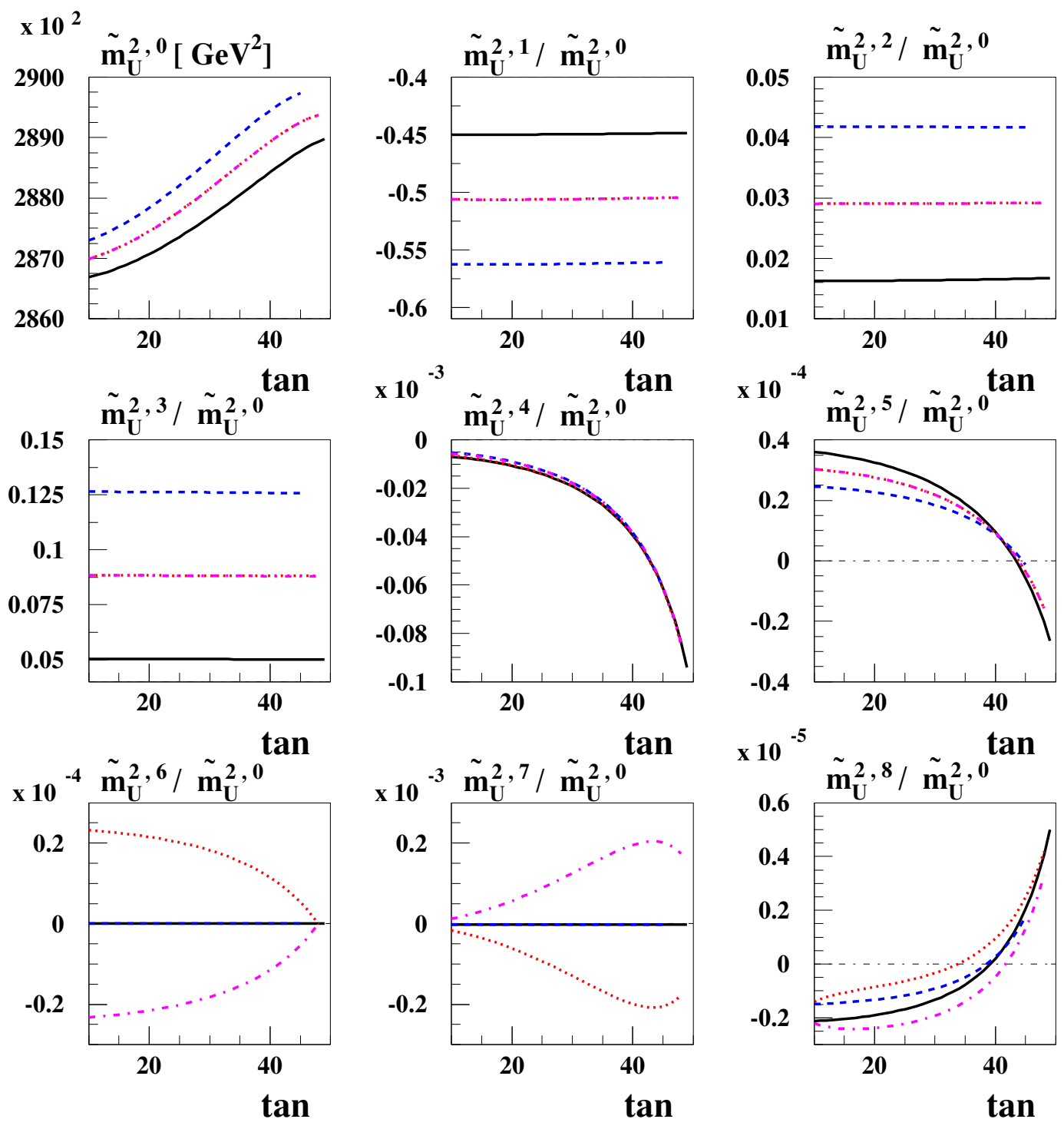

Figure 2. In the top left panel we display the variation of $\widetilde{m}_{U}^{2,0}$ with $\tan \beta$, in the expansion corresponding to (2.5) for the right-handed up-squark mass matrix, as evaluated at the SUSYbreaking scale. In the other panels we display the variations with $\tan \beta$ of the ratios $\widetilde{m}_{U}^{2, I} / \widetilde{m}_{U}^{2,0}$. The lines are the same as in figure 1. The input MCPMFV SUSY-breaking parameters are taken as in (2.17).

9-dimensional flavour-space. As before, we observe a stronger $\tan \beta$-dependence amongst the coefficients of $\mathbf{a}_{d}$ than $\mathbf{a}_{u}$. We note that only the $I=0,2$ coefficients of $\mathbf{a}_{\mathbf{u}, \mathbf{d}}$ share the tendency of the sfermion mass matrices to exhibit the largest RGE effects at $\Phi_{M}=180^{\circ}$, with this behaviour being inverted amongst the remaining coefficients. 

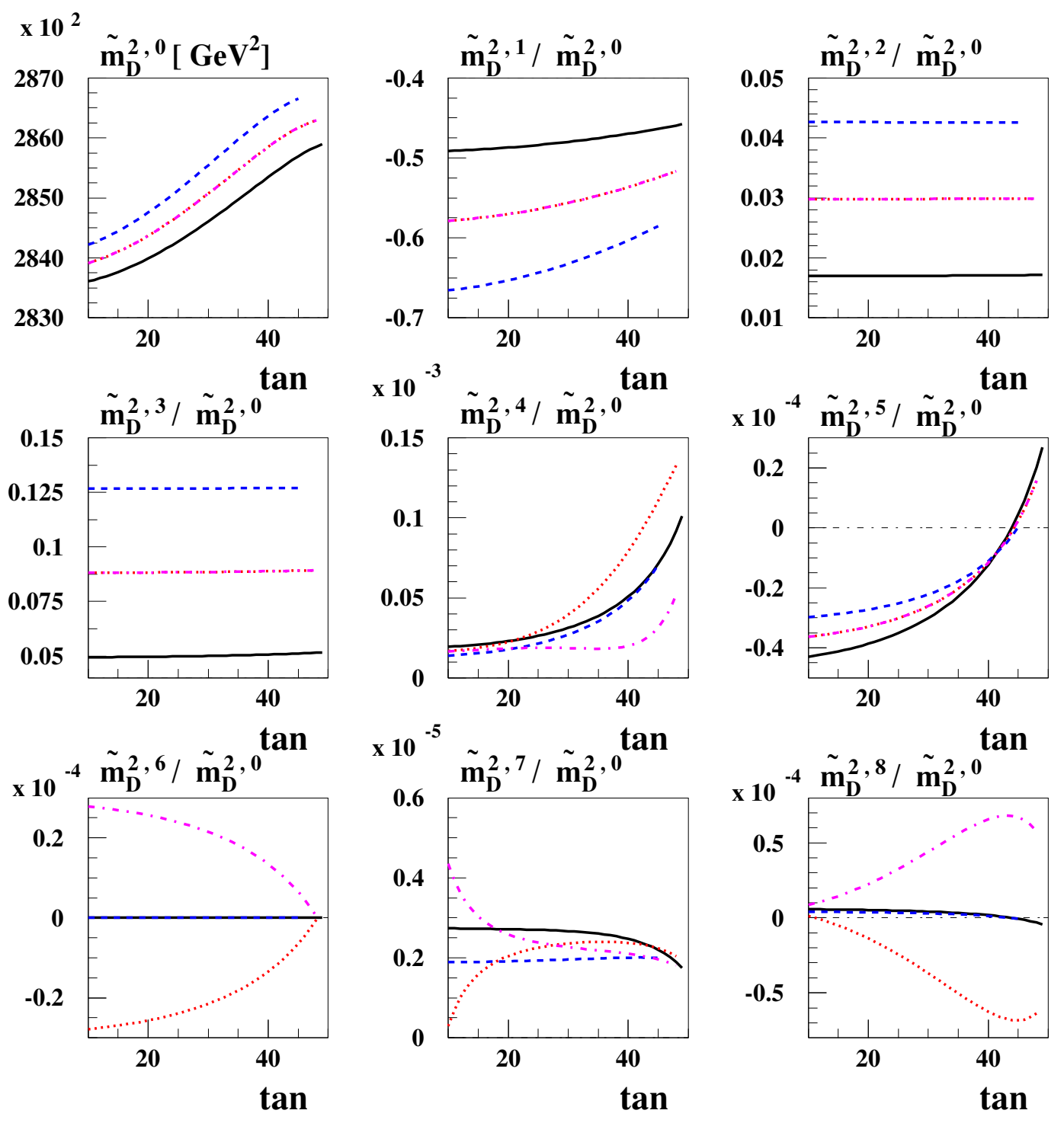

Figure 3. In the top left panel we display the variation of $\widetilde{m}_{D}^{2,0}$ with $\tan \beta$, in the expansion corresponding to (2.5) for the right-handed down-squark mass matrix, as evaluated at the SUSYbreaking scale. In the other panels we display the variations with $\tan \beta$ of the $\operatorname{ratios} \widetilde{m}_{D}^{2, I} / \widetilde{m}_{D}^{2,0}$. The lines are the same as in figure 1. The input MCPMFV SUSY-breaking parameters are taken as in (2.17).

\section{Effective Yukawa couplings at large $\tan \beta$}

In this section we discuss the one-loop threshold corrections to the Yukawa couplings of the MSSM Higgs bosons to fermions. We include explicitly the contributions due to flavourchanging structure in the soft SUSY-breaking terms. Following the flavour-covariant approach of $[15,33]$, we first present the calculation of these effects in the weak basis for the down-type quarks, up-type quarks and leptons, and then relate these to the relevant couplings in the mass eigenbasis. Finally, we present representative numerical results for 

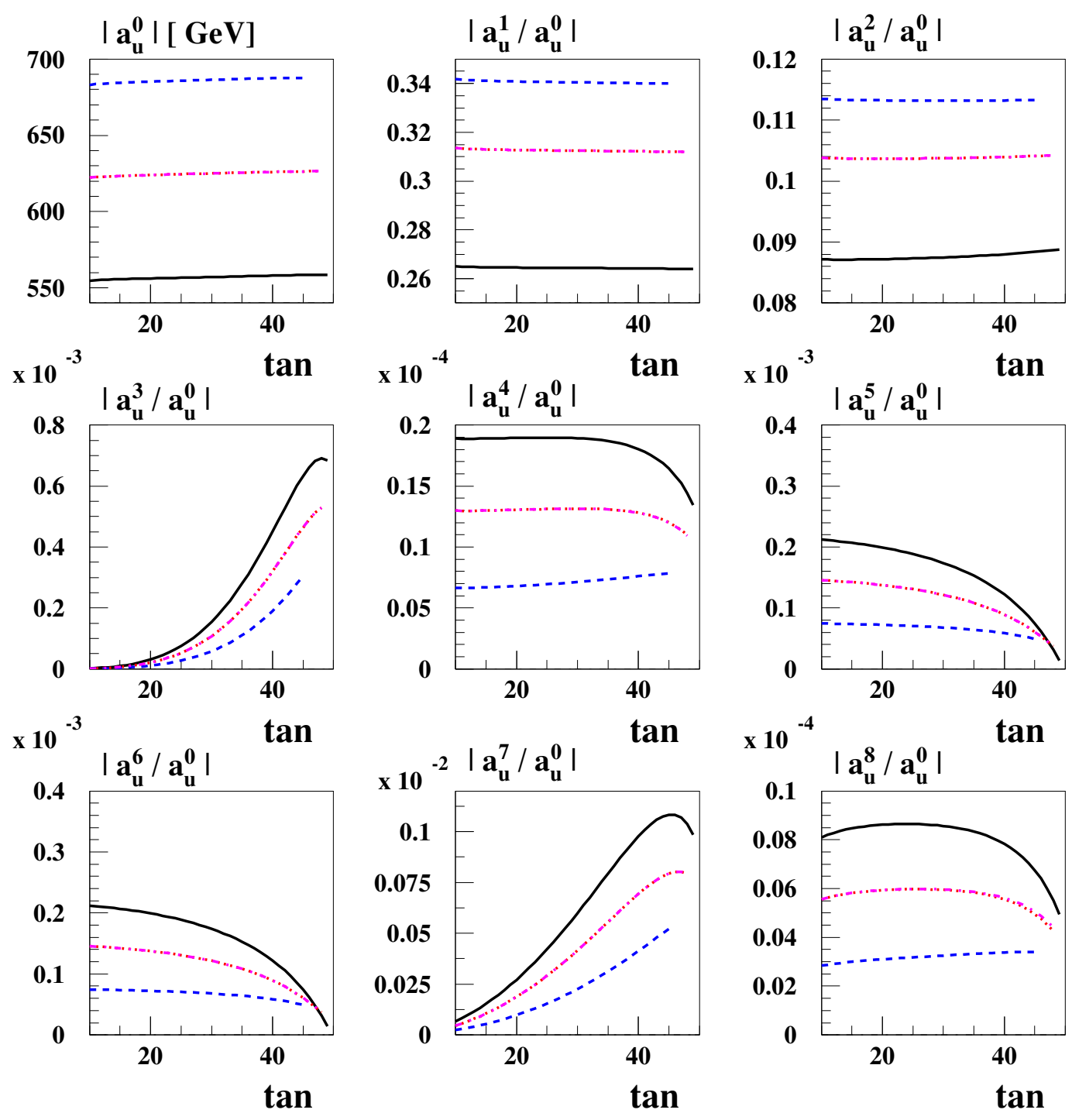

Figure 4. In the top left panel we display the variation of $a_{u}^{0}$ with $\tan \beta$, in the expansion for the trilinear coupling $\mathbf{a}_{u}$ in (2.14), as evaluated at the SUSY-breaking scale. In the other panels we display the variations with $\tan \beta$ of the ratios $\left|a_{u}^{I} / a_{u}^{0}\right|$. The lines are the same as in figure 1 . The input MCPMFV SUSY-breaking parameters are taken as in (2.17).

the Higgs-boson FCNC couplings that can be relevant for the $B_{d, s^{-}}$and $K$-meson systems.

\subsection{Threshold corrections to Yukawa couplings}

\subsubsection{Down-type Quark Yukawa couplings}

In this section we present the complete set of one-loop SUSY corrections to the self-energies of the down-type quarks. These may be described by the effective Lagrangian

$$
-\mathcal{L}_{\text {eff }}^{d}\left[\Phi_{1}, \Phi_{2}\right]=\bar{d}_{i R}^{0}\left(\mathbf{h}_{d} \Phi_{1}^{\dagger \alpha}+\Delta \mathbf{h}_{d}^{\alpha}\left[\Phi_{1}, \Phi_{2}\right]\right)_{i j} Q_{j L}^{0 \alpha},
$$



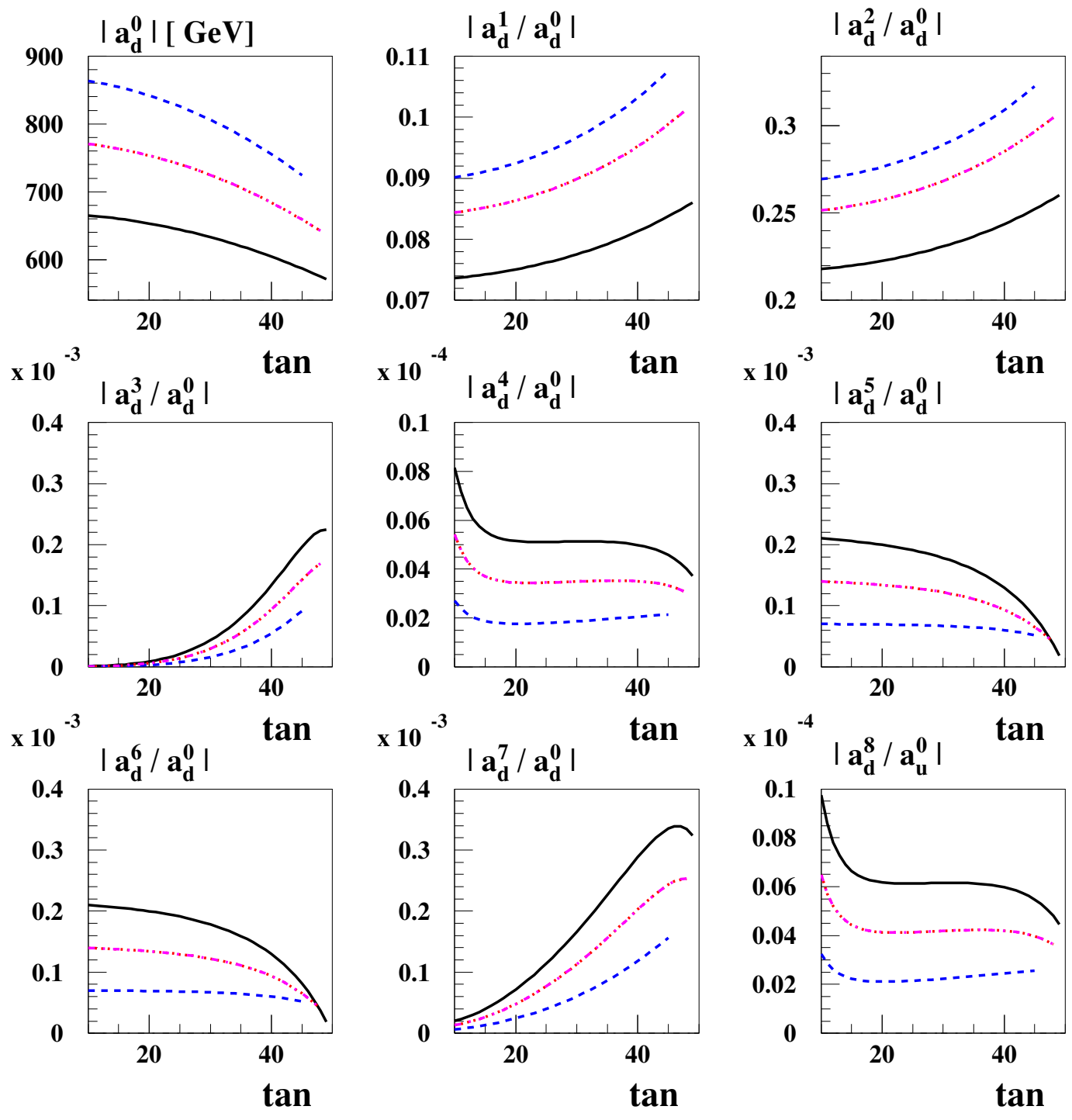

Figure 5. As in figure 4, but for variations of the coefficients $a_{d}^{I}$ with $\tan \beta$, in the expansion for the trilinear coupling $\mathbf{a}_{d}$ in (2.14).

where $\alpha=1,2$ is a weak-isospin index and $\Phi_{1(2)}$ are the scalar components of the Higgs doublet superfields giving masses to the down-type (up-type) quarks respectively. ${ }^{2}$ In (3.1) the first term gives the tree-level contribution, whilst $\Delta \mathbf{h}_{d}$ is a $3 \times 3$ matrix which is a Coleman-Weinberg type effective functional of the background Higgs fields [35], which we decompose as

$$
\boldsymbol{\Delta} \mathbf{h}_{d}=\boldsymbol{\Delta} \mathbf{h}_{d}^{2 \mathrm{HDM}}+\boldsymbol{\Delta} \mathbf{h}_{d}^{\mathrm{SUSY}}+\boldsymbol{\Delta} \mathbf{h}_{d}^{\mathrm{CT}},
$$

\footnotetext{
${ }^{2}$ Here we adopt the convention for the Higgs doublets: $H_{u} \equiv \Phi_{2}, H_{d} \equiv i \sigma_{2} \Phi_{1}^{*}$, where $\sigma_{2}$ is the usual Pauli matrix.
} 
where $\boldsymbol{\Delta} \mathbf{h}_{d}^{\mathrm{CT}}$ contains the counterterms required to cancel the divergences of the first two terms. The contributions $\boldsymbol{\Delta} \mathbf{h}_{d}^{2 \mathrm{HDM}}$ are insensitive to the flavour structure of the soft-SUSY breaking mass and trilinear matrices at the one-loop level and, unless explicitly stated, we neglect them in what follows. Due to the no-renormalization theorem for the SUSY superpotential, the MSSM Yukawa couplings are renormalized only by the wave-function counterterms $Z_{\hat{D}, \hat{H}_{1}, \hat{Q}}^{1 / 2}$ of the Higgs and quark superfields, so that

$$
\Delta \mathbf{h}_{d}^{\mathrm{CT}}=\left(Z_{\hat{D}}^{\frac{1}{2}} Z_{\hat{H}_{1}}^{\frac{1}{2}} Z_{\hat{Q}}^{\frac{1}{2}}-1\right) \mathbf{h}_{d} \Phi_{1}^{\dagger} \simeq \frac{1}{2} \sum_{i=\hat{D}, \hat{H}_{1}, \hat{Q}} \delta Z_{i} \mathbf{h}_{d} \Phi_{1}^{\dagger}
$$

with $\delta Z_{i}=Z_{i}-1$. Note that there are no counterterms to the down-type quark Yukawa coupling proportional to $\Phi_{2}^{\dagger}$.

The one-loop SUSY threshold corrections to the down-type quark self-energy may be calculated from the Feynman diagrams of figure 6. More explicitly, these are given by

$$
\begin{aligned}
& -\left(\Delta \mathbf{h}_{d}^{\text {SUSY }}\right)_{i j}^{\alpha}=\int \frac{d^{n} k}{(2 \pi)^{n} i}\left[P_{L} \frac{-2 C_{F} g_{3}^{2} M_{3}^{*}}{k^{2}-\left|M_{3}^{2}\right|}\left(\frac{1}{k^{2} \mathbf{1}_{12}-\widetilde{\mathbf{M}}^{2}}\right)_{\tilde{D}_{i} \tilde{Q}_{j}^{\dagger \alpha}}\right)_{\tilde{B} \tilde{B}} P_{L}\left(\frac{1}{k^{2} \mathbf{1}_{12}-\widetilde{\mathbf{M}}^{2}}\right)_{\tilde{D}_{i} \tilde{Q}_{j}^{\dagger \alpha}} \\
& +P_{L} \frac{g_{1}^{2}}{9}\left(\frac{1}{\not k \mathbf{1}_{8}-\mathbf{M}_{C} P_{L}-\mathbf{M}_{C}^{\dagger} P_{R}}\right)_{\tilde{H}_{d}^{\gamma} \tilde{H}_{u}^{\beta}} P_{L}\left(\mathbf{h}_{d}\right)_{i l}\left(i \sigma_{2}\right)^{\gamma \delta}\left(\frac{1}{k^{2} \mathbf{1}_{12}-\widetilde{\mathbf{M}}^{2}}\right)_{\tilde{Q}_{l}^{\delta} \tilde{U}_{k}^{\dagger}}\left(\mathbf{h}_{u}\right)_{k j}\left(-i \sigma_{2}\right)^{\beta \alpha} \\
& +P_{L}\left(\frac{1}{\not k \mathbf{1}_{8}-\mathbf{M}_{C} P_{L}-\mathbf{M}_{C}^{\dagger} P_{R}}\right)_{\tilde{H}_{d}^{\gamma} \tilde{B}} P_{L}\left(\mathbf{h}_{d}\right)_{i l}\left(i \sigma_{2}\right)^{\gamma \beta}\left(\frac{1}{k^{2} \mathbf{1}_{12}-\widetilde{\mathbf{M}}^{2}}\right)_{\tilde{Q}_{l}^{\beta} \tilde{Q}_{j}^{\dagger \alpha}}\left(\frac{g_{1}}{3 \sqrt{2}}\right) \\
& +\sum_{k} P_{L}\left(\frac{1}{\not k \mathbf{1}_{8}-\mathbf{M}_{C} P_{L}-\mathbf{M}_{C}^{\dagger} P_{R}}\right)_{\tilde{H}_{d}^{\delta} \tilde{W}^{k}} P_{L}\left(\mathbf{h}_{d}\right)_{i l}\left(i \sigma_{2}\right)^{\delta \gamma}\left(\frac{1}{k^{2} \mathbf{1}_{12}-\widetilde{\mathbf{M}}^{2}}\right)_{\tilde{Q}_{l}^{\gamma} \tilde{Q}_{j}^{\dagger \beta}}\left(\frac{g_{2} \sigma_{k}^{\beta \alpha}}{\sqrt{2}}\right) \\
& \left.+P_{L}\left(\frac{1}{\not k \mathbf{1}_{8}-\mathbf{M}_{C} P_{L}-\mathbf{M}_{C}^{\dagger} P_{R}}\right)_{\tilde{B} \tilde{H}_{d}^{\beta}}\left(\frac{2 g_{1}}{3 \sqrt{2}}\right) P_{L}\left(\frac{1}{k^{2} \mathbf{1}_{12}-\widetilde{\mathbf{M}}^{2}}\right)_{\tilde{D}_{i} \tilde{D}_{l}^{\dagger}}\left(\mathbf{h}_{d}\right)_{l j}\left(i \sigma_{2}\right)^{\beta \alpha}\right],
\end{aligned}
$$

where $\sigma_{k}$ are the usual Pauli matrices, $P_{L(R)}=\frac{1}{2}\left[1-(+) \gamma_{5}\right]$ is the left-handed (righthanded) chiral projection operator and $g_{1,2,3}$ are the gauge coupling constants of $\mathrm{U}(1)_{Y}, \mathrm{SU}(2)_{L}$ and $\mathrm{SU}(3)_{c}$, respectively. Also, $C_{F}$ is the quadratic Casimir invariant of QCD in the fundamental representation, i.e., $C_{F}=4 / 3$, and $\mathbf{1}_{N}$ is the $N \times N$ identity matrix. The $12 \times 12$ squark mass-squared matrix $\widetilde{\mathbf{M}}^{2}$ and the $8 \times 8$ chargino-neutralino mass matrix $\mathbf{M}_{C}$ are given in appendix A. The Greek superscripts in (3.4) represent $\mathrm{SU}(2)_{L}$ indices.

We may obtain useful approximations to (3.4) by expanding in powers of the Higgs field $\Phi_{1,2}$, so that

$$
\boldsymbol{\Delta}_{d} \simeq \mathbf{h}_{d}\left\langle\boldsymbol{\Delta}_{d}^{\Phi_{1}}\right\rangle_{0} \Phi_{1}^{\dagger}+\mathbf{h}_{d}\left\langle\boldsymbol{\Delta}_{d}^{\Phi_{2}}\right\rangle_{0} \Phi_{2}^{\dagger}+\ldots
$$




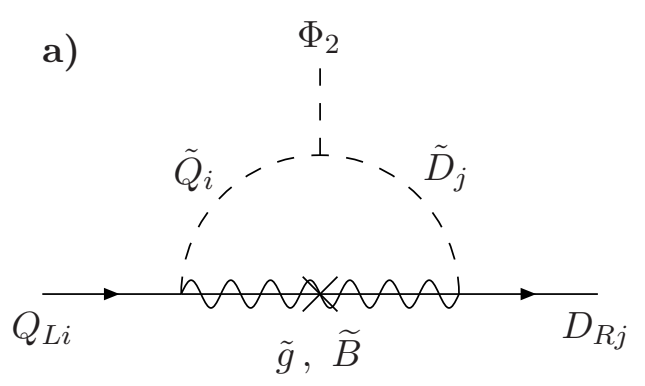

c)

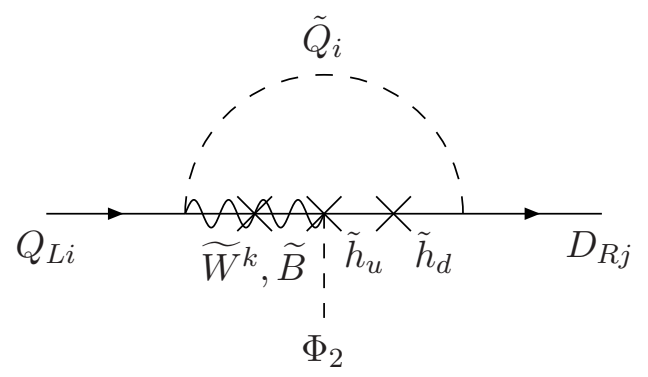

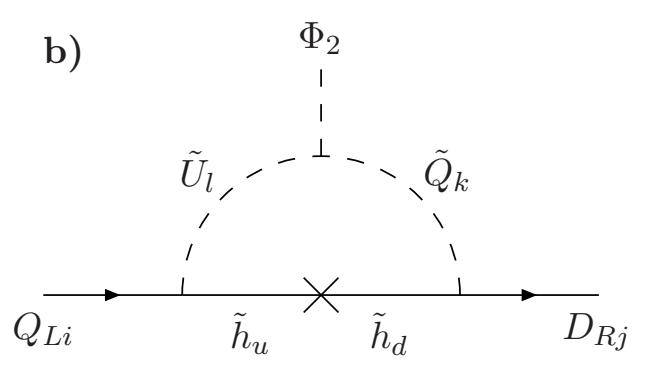

d)

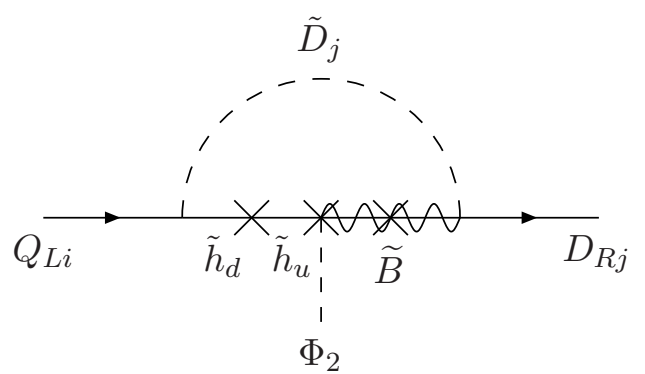

Figure 6. The complete set of gauge- and flavour-covariant one-loop diagrams contributing to the down-type quark self-energy, to first order in $\Phi_{2}$. Note that in panels (a) and (c) contributions from all listed gauginos should be included.

where $\langle\ldots\rangle_{0}$ indicates the value setting all background fields to zero in the expression enclosed, and we have introduced

$$
\boldsymbol{\Delta}_{d}^{\Phi_{i}} \equiv \mathbf{h}_{d}^{-1} \frac{\delta \boldsymbol{\Delta} \mathbf{h}_{d}}{\delta \Phi_{i}^{\dagger}}
$$

The higher-order terms correspond to Feynman diagrams with additional Higgs insertions along the internal propagators, which are typically suppressed by additional factors of $\left(M_{\mathrm{EW}} / M_{\mathrm{SUSY}}\right)^{2}$ and may safely be neglected. We call the first term of such an expansion the "single-Higgs-insertion" (SHI) approximation.

Working in the SHI approximation and assuming flavour-diagonal soft SUSY-breaking terms, (3.4) may be written using the expansion (3.5) in the form

$$
\begin{aligned}
\left\langle\boldsymbol{\Delta}_{d}^{\Phi_{2}}\right\rangle_{0}= & \mathbf{1} \frac{2 \alpha_{3}}{3 \pi} \mu^{*} M_{3}^{*} I\left(\widetilde{M}_{Q}^{2}, \widetilde{M}_{D}^{2},\left|M_{3}\right|^{2}\right)-\mathbf{1} \frac{\alpha_{1}}{36 \pi} \mu^{*} M_{1}^{*} I\left(\widetilde{M}_{Q}^{2}, \widetilde{M}_{D}^{2},\left|M_{1}\right|^{2}\right) \\
& +\frac{\mathbf{h}_{u}^{\dagger} \mathbf{h}_{u}}{16 \pi^{2}} \mu^{*} A_{u}^{*} I\left(\widetilde{M}_{Q}^{2}, \widetilde{M}_{U}^{2},|\mu|^{2}\right)-\mathbf{1} \frac{3 \alpha_{2}}{8 \pi} \mu^{*} M_{2}^{*} I\left(\widetilde{M}_{Q}^{2},\left|M_{2}\right|^{2},|\mu|^{2}\right) \\
& -\mathbf{1} \frac{\alpha_{1}}{24 \pi} \mu^{*} M_{1}^{*} I\left(\widetilde{M}_{Q}^{2},\left|M_{1}\right|^{2},|\mu|^{2}\right)-\mathbf{1} \frac{\alpha_{1}}{12 \pi} \mu^{*} M_{1}^{*} I\left(\widetilde{M}_{D}^{2},\left|M_{1}\right|^{2},|\mu|^{2}\right),
\end{aligned}
$$




$$
\begin{aligned}
\left\langle\boldsymbol{\Delta}_{d}^{\Phi_{1}}\right\rangle_{0}= & -\mathbf{1} \frac{2 \alpha_{3}}{3 \pi} A_{d} M_{3}^{*} I\left(\widetilde{M}_{Q}^{2}, \widetilde{M}_{D}^{2},\left|M_{3}\right|^{2}\right)+\mathbf{1} \frac{\alpha_{1}}{36 \pi} A_{d} M_{1}^{*} I\left(\widetilde{M}_{Q}^{2}, \widetilde{M}_{D}^{2},\left|M_{1}\right|^{2}\right) \\
& -\frac{\mathbf{h}_{u}^{\dagger} \mathbf{h}_{u}}{16 \pi^{2}}|\mu|^{2} I\left(\widetilde{M}_{Q}^{2}, \widetilde{M}_{U}^{2},|\mu|^{2}\right)+\mathbf{1} \frac{3 \alpha_{2}}{8 \pi} B_{0}\left(0,\left|M_{2}\right|^{2}, M_{Q}^{2}\right) \\
& +\mathbf{1} \frac{\alpha_{1}}{24 \pi} B_{0}\left(0,\left|M_{1}\right|^{2}, M_{Q}^{2}\right)+\mathbf{1} \frac{\alpha_{1}}{12 \pi} B_{0}\left(0,\left|M_{1}\right|^{2}, M_{D}^{2}\right),
\end{aligned}
$$

where $\mathbf{1} \equiv \mathbf{1}_{3}$ and $\alpha_{i}=g_{i}^{2} / 4 \pi$ as usual. In writing down (3.7) and (3.8), we have made use of the the one-loop function $I(a, b, c)$, given by

$$
I(a, b, c)=\frac{a b \ln (a / b)+b c \ln (b / c)+a c \ln (c / a)}{(a-b)(b-c)(a-c)},
$$

and the Passarino-Veltman function $B_{0}(p, a, b)$, which may be written as

$$
B_{0}(0, a, b)=1-\ln \left(\frac{b}{Q^{2}}\right)+\frac{a}{a-b} \ln \left(\frac{b}{a}\right),
$$

when the first argument $p^{2}$ is set to zero, i.e. $p^{2}=0$. Here $Q^{2}$ is the renormalisation scale. The approximation (3.7) agrees with other results from the literature $[27,36]$ in the appropriate limits.

In both (3.4) and the approximate expressions (3.7) and (3.8), the first two contributions are the gluino- and bino-mediated corrections of the form shown in figure 6(a), whereas the third term represents the charged Higgsino diagram of figure 6(b). We note that under the assumption of Minimal Flavour Violation, this Higgsino term is the only correction with a non-trivial flavour structure at the one-loop level. The fourth and fifth terms give, respectively, the contributions due to wino- and bino- exchange diagrams of the type shown in figure 6(c), whereas the final term is due to the bino-exchange diagram of figure $6(\mathrm{~d})$. We note that this final contribution is the only one that is independent of the soft SUSY-breaking left-handed squark mass, $\widetilde{M}_{Q}^{2}$.

However, as discussed in section 2.1, the RG running of the soft-SUSY breaking parameters provides an additional source for flavour violation. To leading order in the shift parameters $\boldsymbol{\delta} \widetilde{\mathbf{M}}_{Q, U, D}^{2}$ and $\boldsymbol{\delta} \mathbf{a}_{u, d}$ given in (2.16), ${ }^{3}$ we find that the threshold corrections (3.7) and (3.8) are modified by amounts

$$
\begin{aligned}
&\langle\boldsymbol{\delta}\left.\boldsymbol{\Delta}_{d}^{\Phi_{2}}\right\rangle_{0}=\frac{2 \alpha_{3}}{3 \pi} \mu^{*} M_{3}^{*}\left[\boldsymbol{\delta} \widetilde{\mathbf{M}}_{Q}^{2} K\left(\widetilde{M}_{Q}^{2}, \widetilde{M}_{D}^{2},\left|M_{3}\right|^{2}\right)+\mathbf{h}_{d}^{-1} \boldsymbol{\delta} \widetilde{\mathbf{M}}_{D}^{2} \mathbf{h}_{d} K\left(\widetilde{M}_{D}^{2}, \widetilde{M}_{Q}^{2},\left|M_{3}\right|^{2}\right)\right] \\
&-\frac{\alpha_{1}}{36 \pi} \mu^{*} M_{1}^{*}\left[\boldsymbol{\delta} \widetilde{\mathbf{M}}_{Q}^{2} K\left(\widetilde{M}_{Q}^{2}, \widetilde{M}_{D}^{2},\left|M_{1}\right|^{2}\right)+\mathbf{h}_{d}^{-1} \boldsymbol{\delta} \widetilde{\mathbf{M}}_{D}^{2} \mathbf{h}_{d} K\left(\widetilde{M}_{D}^{2}, \widetilde{M}_{Q}^{2},\left|M_{1}\right|^{2}\right)\right] \\
&+\frac{1}{16 \pi^{2}} \mu^{*} A_{u}^{*}\left[\mathbf{h}_{u}^{\dagger} \boldsymbol{\delta} \widetilde{\mathbf{M}}_{U}^{2} \mathbf{h}_{u} K\left(\widetilde{M}_{U}^{2}, \widetilde{M}_{Q}^{2},|\mu|^{2}\right)+\boldsymbol{\delta} \widetilde{\mathbf{M}}_{Q}^{2} \mathbf{h}_{u}^{\dagger} \mathbf{h}_{u} K\left(\widetilde{M}_{Q}^{2}, \widetilde{M}_{U}^{2},|\mu|^{2}\right)\right] \\
&+\frac{\boldsymbol{\delta} \mathbf{a}_{u}^{\dagger} \mathbf{h}_{u}}{16 \pi^{2}} \mu^{*} I\left(\widetilde{M}_{Q}^{2}, \widetilde{M}_{U}^{2},|\mu|^{2}\right)-\frac{3 \alpha_{2}}{8 \pi} \mu^{*} M_{2}^{*} \boldsymbol{\delta} \widetilde{\mathbf{M}}_{Q}^{2} K\left(\widetilde{M}_{Q}^{2},\left|M_{2}\right|^{2},|\mu|^{2}\right) \\
&-\frac{\alpha_{1}}{24 \pi} \mu^{*} M_{1}^{*} \boldsymbol{\delta} \widetilde{\mathbf{M}}_{Q}^{2} K\left(\widetilde{M}_{Q}^{2},\left|M_{1}\right|^{2},|\mu|^{2}\right)-\frac{\alpha_{1}}{12 \pi} \mu^{*} M_{1}^{*} \mathbf{h}_{d}^{-1} \boldsymbol{\delta} \widetilde{\mathbf{M}}_{D}^{2} \mathbf{h}_{d} K\left(\widetilde{M}_{D}^{2},\left|M_{1}\right|^{2},|\mu|^{2}\right) \\
&\left\langle\boldsymbol{\delta} \boldsymbol{\Delta}_{d}^{\Phi_{1}}\right\rangle_{0}=-\frac{2 \alpha_{3}}{3 \pi} A_{d} M_{3}^{*}\left[\delta \widetilde{\mathbf{M}}_{Q}^{2} K\left(\widetilde{M}_{Q}^{2}, \widetilde{M}_{D}^{2},\left|M_{3}\right|^{2}\right)+\mathbf{h}_{d}^{-1} \boldsymbol{\delta} \widetilde{\mathbf{M}}_{D}^{2} \mathbf{h}_{d} K\left(\widetilde{M}_{D}^{2}, \widetilde{M}_{Q}^{2},\left|M_{3}\right|^{2}\right)\right] \\
& \quad-\frac{2 \alpha_{3}}{3 \pi} \mathbf{h}_{d}^{-1} \boldsymbol{\delta} \mathbf{a}_{d} M_{3}^{*} I\left(\widetilde{M}_{Q}^{2}, \widetilde{M}_{D}^{2},\left|M_{3}\right|^{2}\right)+\frac{\alpha_{1}}{36 \pi} \mathbf{h}_{d}^{-1} \boldsymbol{\delta} \mathbf{a}_{d} M_{1}^{*} I\left(\widetilde{M}_{Q}^{2}, \widetilde{M}_{D}^{2},\left|M_{1}\right|^{2}\right)
\end{aligned}
$$

\footnotetext{
${ }^{3}$ A similar expansion, albeit non-flavour covariant, was also considered in [37].
} 
a)

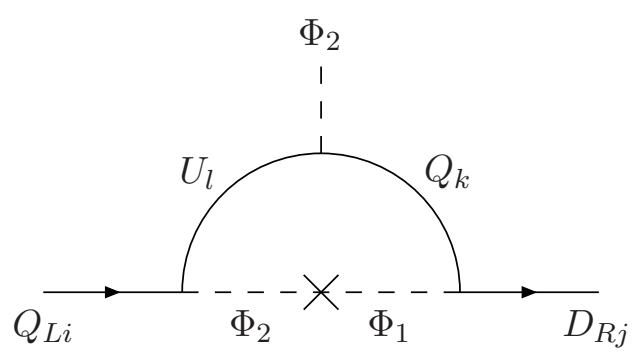

b)

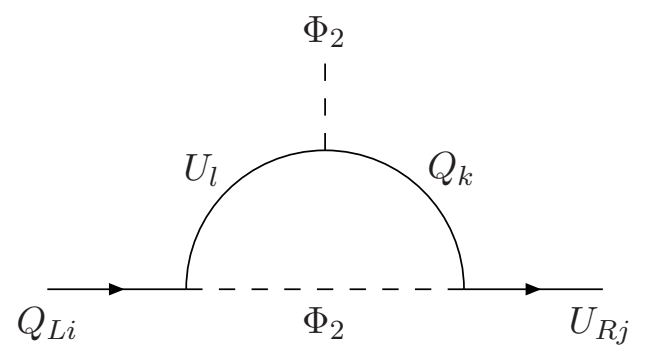

Figure 7. $2 \mathrm{HDM}$ contribution to the down-type (a) and up-type (b) quark self-energies, to first order in $\Phi_{2}$.

$$
\begin{aligned}
& +\frac{\alpha_{1}}{36 \pi} A_{d} M_{1}^{*}\left[\delta \widetilde{\mathbf{M}}_{Q}^{2} K\left(\widetilde{M}_{Q}^{2}, \widetilde{M}_{D}^{2},\left|M_{1}\right|^{2}\right)+\mathbf{h}_{d}^{-1} \boldsymbol{\delta} \widetilde{\mathbf{M}}_{D}^{2} \mathbf{h}_{d} K\left(\widetilde{M}_{D}^{2}, \widetilde{M}_{Q}^{2},\left|M_{1}\right|^{2}\right)\right] \\
& -\frac{1}{16 \pi^{2}}|\mu|^{2}\left[\mathbf{h}_{u}^{\dagger} \boldsymbol{\delta} \widetilde{\mathbf{M}}_{U}^{2} \mathbf{h}_{u} K\left(\widetilde{M}_{U}^{2}, \widetilde{M}_{Q}^{2},|\mu|^{2}\right)+\boldsymbol{\delta} \widetilde{\mathbf{M}}_{Q}^{2} \mathbf{h}_{u}^{\dagger} \mathbf{h}_{u} K\left(\widetilde{M}_{Q}^{2}, \widetilde{M}_{U}^{2},|\mu|^{2}\right)\right] \\
& +\frac{3 \alpha_{2}}{8 \pi} \boldsymbol{\delta} \widetilde{\mathbf{M}}_{Q}^{2} I\left(\widetilde{M}_{Q}^{2},\left|M_{2}\right|^{2}\right)+\frac{\alpha_{1}}{24 \pi}\left[\delta \widetilde{\mathbf{M}}_{Q}^{2} I\left(\widetilde{M}_{Q}^{2},\left|M_{1}\right|^{2}\right)+2 \mathbf{h}_{d}^{-1} \boldsymbol{\delta} \widetilde{\mathbf{M}}_{D}^{2} \mathbf{h}_{d} I\left(\widetilde{M}_{D}^{2},\left|M_{1}\right|^{2}\right)\right]
\end{aligned}
$$

where

$$
\begin{aligned}
K(a, b, c) & =\frac{d}{d a} I(a, b, c) \\
& =\frac{b \ln (a / b)+c \ln (c / a)}{(a-b)(b-c)(a-c)}+\frac{(b+c-2 a) I(a, b, c)+1}{(a-b)(a-c)}
\end{aligned}
$$

and

$$
\begin{aligned}
I(a, b) & \equiv \frac{d}{d a} B_{0}(0, a, b)=-\lim _{c \rightarrow a} I(a, b, c) \\
& =\frac{1}{a-b}\left[\frac{b}{a-b} \ln \left(\frac{a}{b}\right)-1\right] .
\end{aligned}
$$

There is also a two-Higgs-doublet model (2HDM) contribution to the one-loop self-energy graphs for down-type quarks which is formally $\tan \beta$-enhanced. This contribution, displayed in figure 7(a), is not affected by flavour non-universal RG effects of the sort discussed above. It may be calculated by evaluating

$$
\begin{aligned}
\left(\Delta \mathbf{h}_{d}^{2 \mathrm{HDM}}\right)_{i j}= & \int \frac{d^{n} k}{(2 \pi)^{n} i}\left(\mathbf{h}_{\mathbf{d}}\right)_{i l} P_{L}\left(\frac{1}{\not k \mathbf{1}_{6}-\mathbf{M}_{q} P_{L}-\mathbf{M}_{q}^{\dagger} P_{R}}\right)_{Q_{l} u_{k}} P_{L}\left(\mathbf{h}_{u}\right)_{k j} \\
& \times\left(\frac{1}{k^{2} \mathbf{1}_{4}-\mathbf{M}_{H}^{2}}\right)_{\Phi_{1} \Phi_{2}^{\dagger}}
\end{aligned}
$$

where $\mathbf{M}_{q}$ is the $6 \times 6$ quark mass matrix and $\mathbf{M}_{H}^{2}$ the $8 \times 8$ Higgs mass-squared matrix. Explicit forms for these matrices are given in appendix A. In the SHI approximation, the 
2HDM contribution of (3.15) may be written as

$$
\left(\boldsymbol{\Delta}_{d}^{\Phi_{2}}\right)^{2 \mathrm{HDM}}=\frac{\mathbf{h}_{u}^{\dagger} \mathbf{h}_{u}}{16 \pi^{2}} \frac{B^{*} \mu^{*}}{M_{H_{d}}^{2}-M_{H_{u}}^{2}} \ln \left|\frac{M_{H_{d}}^{2}+|\mu|^{2}}{M_{H_{u}}^{2}+|\mu|^{2}}\right| .
$$

The corresponding term $\left(\boldsymbol{\Delta}_{d}^{\Phi_{1}}\right)^{2 \mathrm{HDM}}$ for $\Phi_{1}$ is zero at this level of approximation.

\subsubsection{Up-type Quark Yukawa couplings}

We now turn our attention to the up-quark sector. The up-type quark self-energy is described by the effective Lagrangian

$$
-\mathcal{L}_{\text {eff }}^{u}\left[\Phi_{1}, \Phi_{2}\right]=\bar{u}_{i R}^{0}\left(\mathbf{h}_{u} \Phi_{2}^{T \alpha}+\Delta \mathbf{h}_{u}^{\alpha}\left[\Phi_{1}, \Phi_{2}\right]\right)_{i j}\left(-i \sigma_{2}\right)^{\alpha \beta} Q_{j L}^{0 \beta},
$$

where

$$
\boldsymbol{\Delta} \mathbf{h}_{u}=\boldsymbol{\Delta} \mathbf{h}_{u}^{2 \mathrm{HDM}}+\boldsymbol{\Delta} \mathbf{h}_{u}^{\mathrm{SUSY}}+\boldsymbol{\Delta} \mathbf{h}_{u}^{\mathrm{CT}},
$$

and $\boldsymbol{\Delta} \mathbf{h}_{u}^{\mathrm{CT}}$ is due to the wave-function renormalization of the Higgs and quark superfields. The corrections to the up-type quark self-energy are given by the set of diagrams displayed in figure 8. We may express these corrections as

$$
\begin{aligned}
& -\left(\Delta \mathbf{h}_{u}^{\mathrm{SUSY}}\right)_{i j}^{\alpha}=\int \frac{d^{n} k}{(2 \pi)^{n} i}\left[P_{L} \frac{-2 C_{F} g_{3}^{2} M_{3}^{*}}{k^{2}-\left|M_{3}^{2}\right|}\left(\frac{1}{k^{2} \mathbf{1}_{12}-\widetilde{\mathbf{M}}^{2}}\right)_{\tilde{U}_{i} \tilde{Q}_{j}^{+\beta}}\left(i \sigma_{2}\right)^{\beta \alpha}\right. \\
& +P_{L}\left(\frac{-2 g_{1}^{2}}{9}\right)\left(\frac{1}{\not \mathbf{1}_{8}-\mathbf{M}_{C} P_{L}-\mathbf{M}_{C}^{\dagger} P_{R}}\right)_{\tilde{B} \tilde{B}} P_{L}\left(\frac{1}{k^{2} \mathbf{1}_{12}-\widetilde{\mathbf{M}}^{2}}\right)_{\tilde{U}_{i} \tilde{Q}_{j}^{\dagger \beta}}\left(i \sigma_{2}\right)^{\beta \alpha} \\
& +P_{L}\left(\frac{1}{\not k \mathbf{1}_{8}-\mathbf{M}_{C} P_{L}-\mathbf{M}_{C}^{\dagger} P_{R}}\right)_{\tilde{H}_{u}^{\gamma} \tilde{H}_{d}^{\alpha}} P_{L}\left(\mathbf{h}_{u}\right)_{i l}\left(i \sigma_{2}\right)^{\gamma \beta}\left(\frac{1}{k^{2} \mathbf{1}_{12}-\widetilde{\mathbf{M}}^{2}}\right)_{\tilde{Q}_{l}^{\beta} \tilde{D}_{k}^{\dagger}}\left(\mathbf{h}_{d}\right)_{k j} \\
& +P_{L}\left(\frac{1}{\not k \mathbf{1}_{8}-\mathbf{M}_{C} P_{L}-\mathbf{M}_{C}^{\dagger} P_{R}}\right)_{\tilde{H}_{u}^{\delta} \tilde{B}} P_{L}\left(\mathbf{h}_{u}\right)_{i l}\left(-i \sigma_{2}\right)^{\delta \gamma}\left(\frac{1}{k^{2} \mathbf{1}_{12}-\widetilde{\mathbf{M}}^{2}}\right)_{\tilde{Q}_{l}^{\gamma} \tilde{Q}_{j}^{\dagger \beta}}\left(\frac{g_{1}}{3 \sqrt{2}}\right)(i \sigma)^{\beta \alpha} \\
& +\sum_{k} P_{L}\left(\frac{1}{\not k \mathbf{1}_{8}-\mathbf{M}_{C} P_{L}-\mathbf{M}_{C}^{\dagger} P_{R}}\right)_{\tilde{H}_{u}^{\epsilon} \tilde{W}^{k}} \\
& P_{L}\left(\mathbf{h}_{u}\right)_{i l}\left(-i \sigma_{2}\right)^{\epsilon \delta}\left(\frac{1}{k^{2} \mathbf{1}_{12}-\widetilde{\mathbf{M}}^{2}}\right)_{\tilde{Q}_{l}^{\delta} \tilde{Q}_{j}^{\dagger \gamma}}\left(\frac{g_{2} \sigma_{k}^{\gamma \beta}}{\sqrt{2}}\right)(i \sigma)^{\beta \alpha} \\
& \left.+P_{L}\left(\frac{1}{\not k \mathbf{1}_{8}-\mathbf{M}_{C} P_{L}-\mathbf{M}_{C}^{\dagger} P_{R}}\right)_{\tilde{B} \tilde{H}_{u}^{\alpha}}\left(\frac{-4 g_{1}}{3 \sqrt{2}}\right) P_{L}\left(\frac{1}{k^{2} \mathbf{1}_{12}-\widetilde{\mathbf{M}}^{2}}\right)_{\tilde{U}_{i} \tilde{U}_{l}^{\dagger}}\left(\mathbf{h}_{u}\right)_{l j}\right] \text {. }
\end{aligned}
$$

Expanding (3.19) in powers of $\Phi_{1,2}$, we may write

$$
\boldsymbol{\Delta} \mathbf{h}_{u} \simeq \mathbf{h}_{u}\left\langle\boldsymbol{\Delta}_{u}^{\Phi_{1}}\right\rangle_{0} \Phi_{1}^{T}+\mathbf{h}_{u}\left\langle\boldsymbol{\Delta}_{u}^{\Phi_{2}}\right\rangle_{0} \Phi_{2}^{T}+\ldots
$$

with

$$
\boldsymbol{\Delta}_{u}^{\Phi_{i}}=\mathbf{h}_{u}^{-1} \frac{\delta \boldsymbol{\Delta} \mathbf{h}_{u}}{\delta \Phi_{i}^{T}}
$$




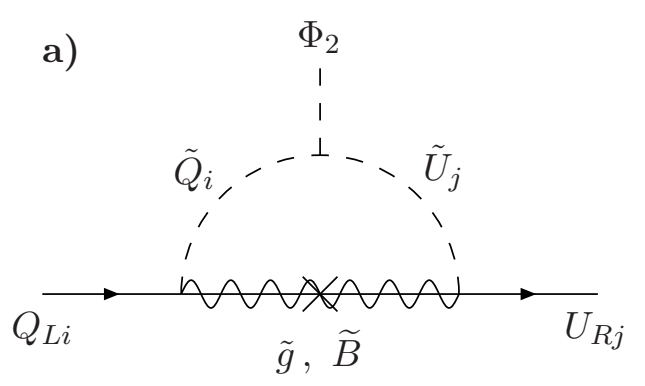

c)

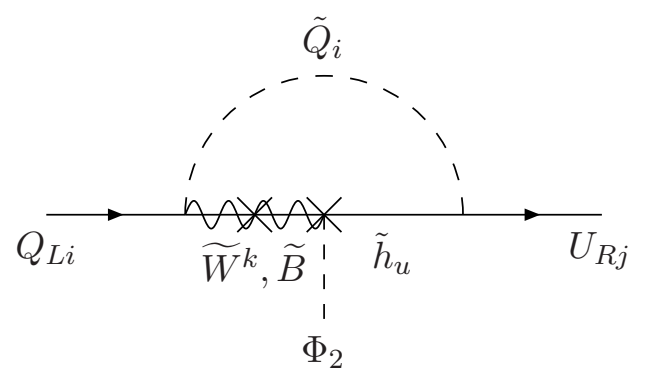

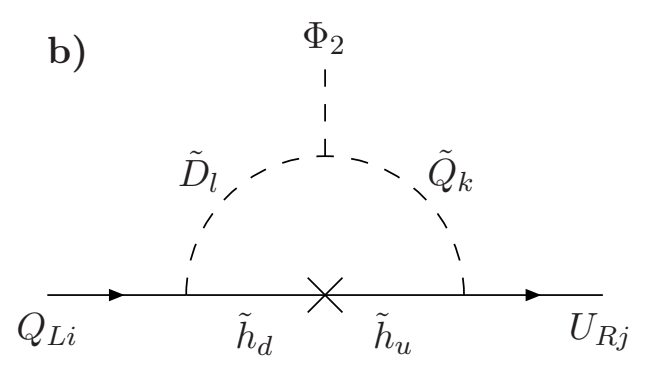

d)

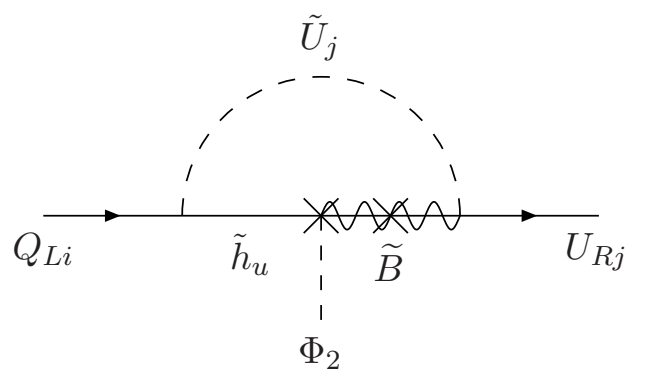

Figure 8. The complete set of gauge- and flavour-covariant diagrams contributing to the up-type quark self-energy, to first order in $\Phi_{2}$. Note that in panels (a) and (c) contributions from all listed gauginos should be included.

so that the up-type quark self-energy is given in the SHI approximation by

$$
\begin{aligned}
\left\langle\boldsymbol{\Delta}_{u}^{\Phi_{2}}\right\rangle_{0}= & -\mathbf{1} \frac{2 \alpha_{3}}{3 \pi} A_{u} M_{3}^{*} I\left(\widetilde{M}_{Q}^{2}, \widetilde{M}_{U}^{2},\left|M_{3}\right|^{2}\right)-\mathbf{1} \frac{\alpha_{1}}{18 \pi} A_{u} M_{1}^{*} I\left(\widetilde{M}_{Q}^{2}, \widetilde{M}_{U}^{2},\left|M_{1}\right|^{2}\right) \\
& -\frac{\mathbf{h}_{d}^{\dagger} \mathbf{h}_{d}}{16 \pi^{2}}|\mu|^{2} I\left(\widetilde{M}_{Q}^{2}, \widetilde{M}_{D}^{2},|\mu|^{2}\right)+\mathbf{1} \frac{3 \alpha_{2}}{8 \pi} B_{0}\left(0, \widetilde{M}_{Q}^{2},\left|M_{2}\right|^{2}\right) \\
& -\mathbf{1} \frac{\alpha_{1}}{24 \pi} B_{0}\left(0,\left|M_{1}\right|^{2}, \widetilde{M}_{Q}^{2}\right)+\mathbf{1} \frac{\alpha_{1}}{6 \pi} B_{0}\left(0,\left|M_{1}\right|^{2}, \widetilde{M}_{U}^{2}\right), \\
\left\langle\boldsymbol{\Delta}_{u}^{\Phi_{1}}\right\rangle_{0}= & \mathbf{1} \frac{2 \alpha_{3}}{3 \pi} \mu^{*} M_{3}^{*} I\left(M_{Q}^{2}, M_{U}^{2},\left|M_{3}\right|^{2}\right)+\mathbf{1} \frac{\alpha_{1}}{18 \pi} \mu^{*} M_{1}^{*} I\left(M_{Q}^{2}, M_{U}^{2},\left|M_{1}\right|^{2}\right) \\
& +\frac{\mathbf{h}_{d}^{\dagger} \mathbf{h}_{d}}{16 \pi^{2}} \mu^{*} A_{d}^{*} I\left(M_{Q}^{2}, M_{D}^{2},|\mu|^{2}\right)-\frac{3 \alpha_{2}}{8 \pi} \mu^{*} M_{2}^{*} I\left(M_{Q}^{2},\left|M_{2}\right|^{2},|\mu|^{2}\right) \\
& +\mathbf{1} \frac{\alpha_{1}}{24 \pi} \mu^{*} M_{1}^{*} I\left(M_{Q}^{2},\left|M_{1}\right|^{2},|\mu|^{2}\right)-\mathbf{1} \frac{\alpha_{1}}{6 \pi} \mu^{*} M_{1}^{*} I\left(M_{U}^{2},\left|M_{1}\right|^{2},|\mu|^{2}\right) .
\end{aligned}
$$

The RG running of the soft-SUSY breaking parameters again provides an additional source of flavour violation. To leading order in the shift parameters $\boldsymbol{\delta} \widetilde{\mathbf{M}}_{Q, U, D}^{2}$ and $\delta \mathbf{a}_{u, d}$ 
given in (2.16), we find that the threshold corrections (3.22) and (3.23) are modified by

$$
\begin{aligned}
&\langle\boldsymbol{\delta}\left.\boldsymbol{\Delta}_{u}^{\Phi_{2}}\right\rangle_{0}=-\frac{2 \alpha_{3}}{3 \pi} A_{u} M_{3}^{*}\left[\boldsymbol{\delta} \widetilde{\mathbf{M}}_{Q}^{2} K\left(\widetilde{M}_{Q}^{2}, \widetilde{M}_{U}^{2},\left|M_{3}\right|^{2}\right)+\mathbf{h}_{u}^{-1} \boldsymbol{\delta} \widetilde{\mathbf{M}}_{U}^{2} \mathbf{h}_{u} K\left(\widetilde{M}_{U}^{2}, \widetilde{M}_{Q}^{2},\left|M_{3}\right|^{2}\right)\right] \\
&-\frac{2 \alpha_{3}}{3 \pi} \mathbf{h}_{u}^{-1} \boldsymbol{\delta} \mathbf{a}_{u} M_{3}^{*} I\left(\widetilde{M}_{Q}^{2}, \widetilde{M}_{U}^{2},\left|M_{3}\right|^{2}\right)-\frac{\alpha_{1}}{18 \pi} \mathbf{h}_{u}^{-1} \boldsymbol{\delta} \mathbf{a}_{u} M_{1}^{*} I\left(\widetilde{M}_{Q}^{2}, \widetilde{M}_{U}^{2},\left|M_{1}\right|^{2}\right) \\
&-\frac{\alpha_{1}}{18 \pi} A_{u} M_{1}^{*}\left[\boldsymbol{\delta} \widetilde{\mathbf{M}}_{Q}^{2} K\left(\widetilde{M}_{Q}^{2}, \widetilde{M}_{U}^{2},\left|M_{1}\right|^{2}\right)+\mathbf{h}_{u}^{-1} \boldsymbol{\delta} \widetilde{\mathbf{M}}_{U}^{2} \mathbf{h}_{u} K\left(\widetilde{M}_{U}^{2}, \widetilde{M}_{Q}^{2},\left|M_{1}\right|^{2}\right)\right] \\
&-\frac{1}{16 \pi^{2}}|\mu|^{2}\left[\mathbf{h}_{d}^{\dagger} \boldsymbol{\delta} \widetilde{\mathbf{M}}_{D}^{2} \mathbf{h}_{d} K\left(\widetilde{M}_{D}^{2}, \widetilde{M}_{Q}^{2},|\mu|^{2}\right)+\boldsymbol{\delta} \widetilde{\mathbf{M}}_{Q}^{2} \mathbf{h}_{d}^{\dagger} \mathbf{h}_{d} K\left(\widetilde{M}_{Q}^{2}, \widetilde{M}_{D}^{2},|\mu|^{2}\right)\right] \\
&+\frac{3 \alpha_{2}}{8 \pi} \boldsymbol{\delta} \widetilde{\mathbf{M}}_{Q}^{2} I\left(\widetilde{M}_{Q}^{2},\left|M_{2}\right|^{2}\right)-\frac{\alpha_{1}}{24 \pi}\left[\boldsymbol{\delta} \widetilde{\mathbf{M}}_{Q}^{2} I\left(\widetilde{M}_{Q}^{2},\left|M_{1}\right|^{2}\right)-4 \mathbf{h}_{u}^{-1} \boldsymbol{\delta} \widetilde{\mathbf{M}}_{U}^{2} \mathbf{h}_{u} I\left(\widetilde{M}_{U}^{2},\left|M_{1}\right|^{2}\right)\right] . \\
&\left\langle\boldsymbol{\delta} \boldsymbol{\Delta}_{u}^{\Phi_{1}}\right\rangle_{0}=\frac{2 \alpha_{3}}{3 \pi} \mu^{*} M_{3}^{*}\left[\boldsymbol{\delta} \widetilde{\mathbf{M}}_{Q}^{2} K\left(\widetilde{M}_{Q}^{2}, \widetilde{M}_{U}^{2},\left|M_{3}\right|^{2}\right)+\mathbf{h}_{u}^{-1} \boldsymbol{\delta} \widetilde{\mathbf{M}}_{U}^{2} \mathbf{h}_{u} K\left(\widetilde{M}_{U}^{2}, \widetilde{M}_{Q}^{2},\left|M_{3}\right|^{2}\right)\right] \\
& \quad+\frac{\alpha_{1}}{18 \pi} \mu^{*} M_{1}^{*}\left[\delta \widetilde{\mathbf{M}}_{Q}^{2} K\left(\widetilde{M}_{Q}^{2}, \widetilde{M}_{U}^{2},\left|M_{1}\right|^{2}\right)+\mathbf{h}_{u}^{-1} \boldsymbol{\delta} \widetilde{\mathbf{M}}_{U}^{2} \mathbf{h}_{u} K\left(\widetilde{M}_{U}^{2}, \widetilde{M}_{Q}^{2},\left|M_{1}\right|^{2}\right)\right] \\
& \quad+\frac{1}{16 \pi^{2}} \mu^{*} A_{d}^{*}\left[\mathbf{h}_{d}^{\dagger} \boldsymbol{\delta} \widetilde{\mathbf{M}}_{D}^{2} \mathbf{h}_{d} K\left(\widetilde{M}_{D}^{2}, \widetilde{M}_{Q}^{2},|\mu|^{2}\right)+\boldsymbol{\delta} \widetilde{\mathbf{M}}_{Q}^{2} \mathbf{h}_{d}^{\dagger} \mathbf{h}_{d} K\left(\widetilde{M}_{Q}^{2}, \widetilde{M}_{D}^{2},|\mu|^{2}\right)\right] \\
&+\frac{\boldsymbol{\delta} \mathbf{a}_{d}^{\dagger} \mathbf{h}_{d}}{16 \pi^{2}} \mu^{*} I\left(\widetilde{M}_{Q}^{2}, \widetilde{M}_{D}^{2},|\mu|^{2}\right)-\frac{3 \alpha_{2}}{8 \pi} \mu^{*} M_{2}^{*} \boldsymbol{\delta} \widetilde{\mathbf{M}}_{Q}^{2} K\left(\widetilde{M}_{Q}^{2},\left|M_{2}\right|^{2},|\mu|^{2}\right) \\
& \quad+\frac{\alpha_{1}}{24 \pi} \mu^{*} M_{1}^{*} \boldsymbol{\delta} \widetilde{\mathbf{M}}_{Q}^{2} K\left(\widetilde{M}_{Q}^{2},\left|M_{1}\right|^{2},|\mu|^{2}\right)-\frac{\alpha_{1}}{6 \pi} \mu^{*} M_{1}^{*} \mathbf{h}_{u}^{-1} \boldsymbol{\delta} \widetilde{\mathbf{M}}_{U}^{2} \mathbf{h}_{u} K\left(\widetilde{M}_{U}^{2},\left|M_{1}\right|^{2},|\mu|^{2}\right) .
\end{aligned}
$$

For completeness, we also display the $2 \mathrm{HDM}$ correction to the up-type quark self-energy analogous to (3.15). This contribution, as shown in figure 7(b), is a Standard Model-like correction which again is not affected by the RG effects discussed above. It is given by

$$
\begin{aligned}
\left(\Delta \mathbf{h}_{u}^{2 \mathrm{HDM}}\right)_{i j}= & \int \frac{d^{n} k}{(2 \pi)^{n} i}\left(\mathbf{h}_{\mathbf{u}}\right)_{i l} P_{L}\left(\frac{1}{\not k \mathbf{1}_{6}-\mathbf{M}_{q} P_{L}-\mathbf{M}_{q}^{\dagger} P_{R}}\right)_{Q_{l} u_{k}} P_{L}\left(\mathbf{h}_{u}\right)_{k j} \\
& \times\left(\frac{1}{k^{2} \mathbf{1}_{4}-\mathbf{M}_{H}^{2}}\right)_{\Phi_{2} \Phi_{2}^{\dagger}}
\end{aligned}
$$

\subsubsection{Lepton Yukawa couplings}

Finally, we discuss the Higgs couplings to the leptonic sector. In analogy to (3.1), we may describe the lepton self-energy by the effective Lagrangian

$$
-\mathcal{L}_{\mathrm{eff}}^{e}\left[\Phi_{1}, \Phi_{2}\right]=\bar{e}_{i R}^{0}\left(\mathbf{h}_{e} \Phi_{1}^{\dagger \alpha}+\Delta \mathbf{h}_{e}^{\alpha}\left[\Phi_{1}, \Phi_{2}\right]\right)_{i j} L_{j L}^{0 \alpha}+\text { H.c. }
$$

where $\boldsymbol{\Delta} \mathbf{h}_{e}$ is a $3 \times 3$ Coleman-Weinberg effective functional of the background Higgs fields which encodes the higher-order corrections. Only the electroweak corrections contribute to the lepton self-energy at the one-loop level, corresponding to the Feynman diagrams shown in figure 9. Again separating out the one-loop counterterms and neglecting the 2HDM-like 


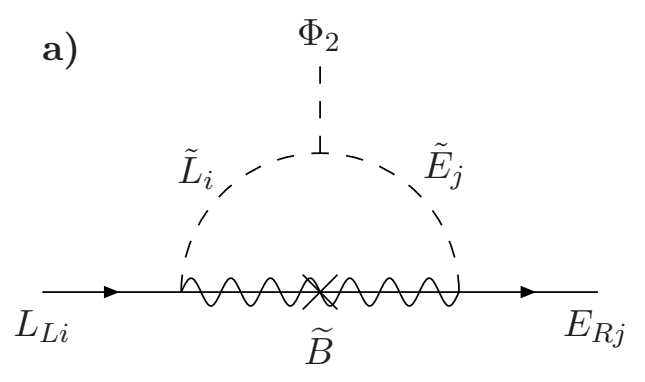

c)

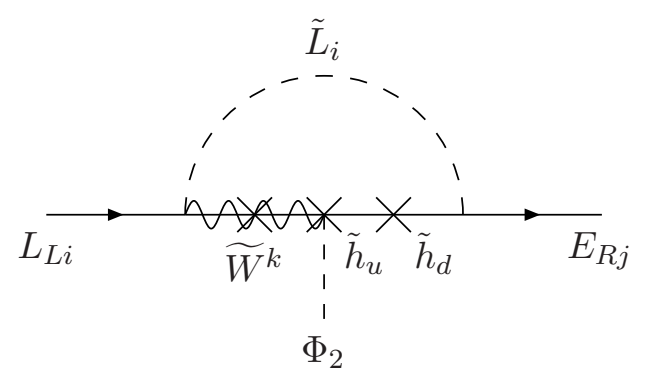

b)

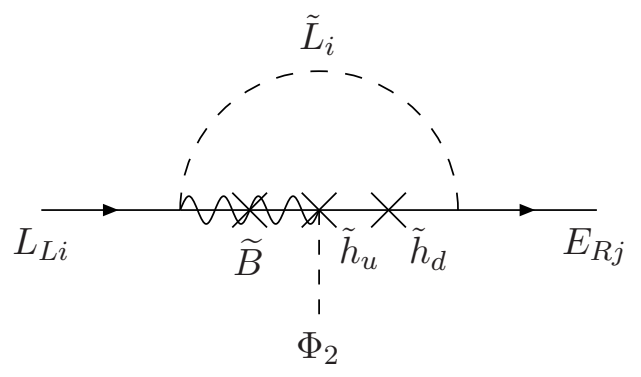

d)

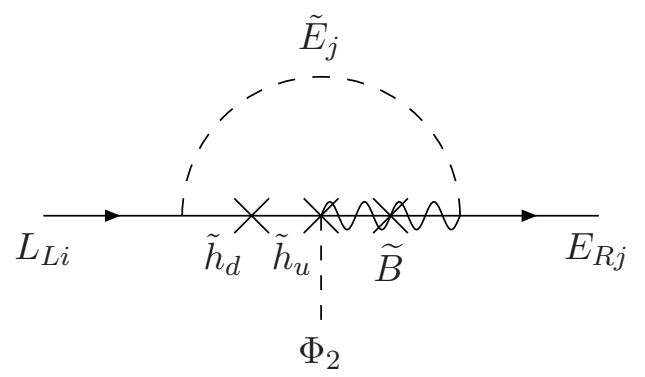

Figure 9. The complete set of gauge- and flavour-covariant diagrams contributing to the lepton self-energy, to first order in $\Phi_{2}$.

contributions, we may write $\Delta \mathbf{h}_{e}^{\mathrm{SUSY}}$ as

$$
\begin{aligned}
& -\left(\boldsymbol{\Delta} \mathbf{h}_{e}^{\mathrm{SUSY}}\right)_{i j}^{\alpha}=\int \frac{d^{n} k}{(2 \pi)^{n} i}\left[-P_{L} g_{1}^{2}\left(\frac{1}{\not k \mathbf{1}_{8}-\mathbf{M}_{C} P_{L}-\mathbf{M}_{C}^{\dagger} P_{R}}\right)_{\tilde{B} \tilde{B}} P_{L}\left(\frac{1}{k^{2} \mathbf{1}_{12}-\widetilde{\mathbf{M}}_{l}^{2}}\right)_{\tilde{E}_{i} \tilde{L}_{j}^{\dagger \alpha}}\right. \\
& +P_{L}\left(\frac{1}{\not k \mathbf{1}_{8}-\mathbf{M}_{C} P_{L}-\mathbf{M}_{C}^{\dagger} P_{R}}\right)_{\tilde{H}_{d}^{\gamma} \tilde{B}} P_{L}\left(\mathbf{h}_{e}\right)_{i l}\left(i \sigma_{2}\right)^{\gamma \beta}\left(\frac{1}{k^{2} \mathbf{1}_{12}-\widetilde{\mathbf{M}}_{l}^{2}}\right)_{\tilde{L}_{l}^{\beta} \tilde{L}_{j}^{\dagger \alpha}}\left(\frac{-g_{1}}{\sqrt{2}}\right) \quad(3.28) \\
& +\sum_{k} P_{L}\left(\frac{1}{\not k \mathbf{1}_{8}-\mathbf{M}_{C} P_{L}-\mathbf{M}_{C}^{\dagger} P_{R}}\right)_{\tilde{H}_{d}^{\delta} \tilde{W}^{k}} P_{L}\left(\mathbf{h}_{e}\right)_{i l}\left(i \sigma_{2}\right)^{\delta \gamma}\left(\frac{1}{k^{2} \mathbf{1}_{12}-\widetilde{\mathbf{M}}_{l}^{2}}\right)_{\tilde{L}_{l}^{\gamma} \tilde{L}_{j}^{\dagger \beta}}\left(\frac{g_{2} \sigma_{k}^{\beta \alpha}}{\sqrt{2}}\right) \\
& \left.+P_{L}\left(\frac{1}{\not k \mathbf{1}_{8}-\mathbf{M}_{C} P_{L}-\mathbf{M}_{C}^{\dagger} P_{R}}\right)_{\tilde{B} \tilde{H}_{d}^{\beta}}\left(\sqrt{2} g_{1}\right) P_{L}\left(\frac{1}{k^{2} \mathbf{1}_{12}-\widetilde{\mathbf{M}}_{l}^{2}}\right)_{\tilde{E}_{i} \tilde{E}_{l}^{\dagger}}\left(\mathbf{h}_{e}\right)_{l j}(i \sigma)^{\beta \alpha}\right],
\end{aligned}
$$

where the $9 \times 9$ slepton mass-squared matrix $\widetilde{\mathbf{M}}_{l}^{2}$ is displayed in appendix A.

Working in the SHI approximation, we may define the quantities $\boldsymbol{\Delta}_{e}^{\Phi_{i}} \equiv \mathbf{h}_{e}^{-1} \boldsymbol{\Delta} \mathbf{h}_{e}^{\Phi_{i}}$ in 
analogy with (3.5). These contributions to the lepton self-energy are given by

$$
\begin{aligned}
\left\langle\boldsymbol{\Delta}_{e}^{\Phi_{2}}\right\rangle_{0}= & \mathbf{1} \frac{\alpha_{1}}{4 \pi} \mu^{*} M_{1}^{*} I\left(\left|M_{1}\right|^{2}, M_{L}^{2}, M_{E}^{2}\right)+\mathbf{1} \frac{\alpha_{1}}{8 \pi} \mu^{*} M_{1}^{*} I\left(\left|M_{1}\right|^{2}, M_{L}^{2},|\mu|^{2}\right) \\
- & \mathbf{1} \frac{3 \alpha_{2}}{8 \pi} \mu^{*} M_{2}^{*} I\left(\left|M_{2}\right|^{2}, M_{L}^{2},|\mu|^{2}\right)-\mathbf{1} \frac{\alpha_{1}}{4 \pi} \mu^{*} M_{1}^{*} I\left(\left|M_{1}\right|^{2}, M_{E}^{2},|\mu|^{2}\right), \\
\left\langle\boldsymbol{\Delta}_{e}^{\Phi_{1}}\right\rangle_{0}= & -\mathbf{1} \frac{\alpha_{1}}{4 \pi} A_{e} M_{1}^{*} I\left(\left|M_{1}\right|^{2}, M_{L}^{2}, M_{E}^{2}\right)-\mathbf{1} \frac{\alpha_{1}}{8 \pi} B_{0}\left(0,\left|M_{1}\right|^{2}, M_{L}^{2}\right) \\
& +\mathbf{1} \frac{3 \alpha_{2}}{8 \pi} B_{0}\left(0,\left|M_{2}\right|^{2}, M_{L}^{2}\right)+\mathbf{1} \frac{\alpha_{1}}{4 \pi} B_{0}\left(0,\left|M_{1}\right|^{2}, M_{E}^{2}\right),
\end{aligned}
$$

and RG running of the soft SUSY-breaking parameters leads to a shift which may be expressed as

$$
\begin{aligned}
\left\langle\delta \boldsymbol{\Delta}_{e}^{\Phi_{2}}\right\rangle_{0}= & \frac{\alpha_{1}}{4 \pi} \mu^{*} M_{1}^{*}\left[\delta \widetilde{\mathbf{M}}_{L}^{2} K\left(\widetilde{\mathbf{M}}_{L}^{2}, \widetilde{\mathbf{M}}_{E}^{2},\left|M_{1}\right|^{2}\right)+\mathbf{h}_{e}^{-1} \boldsymbol{\delta} \widetilde{\mathbf{M}}_{E}^{2} \mathbf{h}_{e} K\left(\widetilde{\mathbf{M}}_{E}^{2}, \widetilde{\mathbf{M}}_{L}^{2},\left|M_{1}\right|^{2}\right)\right] \\
& +\frac{\alpha_{1}}{8 \pi} \mu^{*} M_{1}^{*}\left[\delta \widetilde{\mathbf{M}}_{L}^{2} K\left(\widetilde{\mathbf{M}}_{L}^{2},\left|M_{1}\right|^{2},|\mu|^{2}\right)-2 \mathbf{h}_{e}^{\dagger} \boldsymbol{\delta} \widetilde{\mathbf{M}}_{E}^{2} \mathbf{h}_{e} K\left(\widetilde{\mathbf{M}}_{E}^{2},\left|M_{1}\right|^{2},|\mu|^{2}\right)\right] \\
& -\frac{3 \alpha_{2}}{8 \pi} \mu^{*} M_{2}^{*} \delta \widetilde{\mathbf{M}}_{L}^{2} K\left(\widetilde{\mathbf{M}}_{L}^{2},\left|M_{2}\right|^{2},|\mu|^{2}\right), \\
\left\langle\delta \boldsymbol{\Delta}_{e}^{\Phi_{1}}\right\rangle_{0}= & -\frac{\alpha_{1}}{4 \pi} A_{e} M_{1}^{*}\left[\delta \widetilde{\mathbf{M}}_{L}^{2} K\left(\widetilde{\mathbf{M}}_{L}^{2}, \widetilde{\mathbf{M}}_{E}^{2},\left|M_{1}\right|^{2}\right)+\mathbf{h}_{e}^{-1} \boldsymbol{\delta} \widetilde{\mathbf{M}}_{E}^{2} \mathbf{h}_{e} K\left(\widetilde{\mathbf{M}}_{E}^{2}, \widetilde{\mathbf{M}}_{L}^{2},\left|M_{1}\right|^{2}\right)\right] \\
& -\frac{\alpha_{1}}{4 \pi} \delta \mathbf{a}_{e} M_{1}^{*} I\left(\widetilde{\mathbf{M}}_{L}^{2}, \widetilde{\mathbf{M}}_{E}^{2},\left|M_{1}\right|^{2}\right)+\frac{3 \alpha_{2}}{8 \pi} \boldsymbol{\delta} \widetilde{\mathbf{M}}_{L}^{2} I\left(\widetilde{\mathbf{M}}_{L}^{2},\left|M_{2}\right|^{2}\right) \\
& +\frac{\alpha_{1}}{8 \pi}\left[\delta \widetilde{\mathbf{M}}_{L}^{2} I\left(\widetilde{\mathbf{M}}_{L}^{2},\left|M_{1}\right|^{2}\right)+2 \mathbf{h}_{e}^{-1} \boldsymbol{\delta} \widetilde{\mathbf{M}}_{E}^{2} \mathbf{h}_{e} I\left(\widetilde{\mathbf{M}}_{E}^{2},\left|M_{1}\right|^{2}\right)\right] .
\end{aligned}
$$

where the flavour-non-universal contributions were specified in (2.16).

\subsection{Higgs couplings in the Fermion mass basis}

The weak quark chiral states, $u_{L, R}^{0}$ and $d_{L, R}^{0}$, are related to their respective mass eigenstates, $u_{L, R}$ and $d_{L, R}$, through the unitary transformations:

$$
\begin{aligned}
& u_{L}^{0}=\mathbf{U}_{L}^{Q} u_{L}, \quad d_{L}^{0}=\mathbf{U}_{L}^{Q} \mathbf{V}_{d} d_{L}, \quad u_{R}^{0}=\mathbf{U}_{R}^{u} u_{R}, \quad d_{R}^{0}=\mathbf{U}_{R}^{d} d_{R} \\
& \nu_{L}^{0}=\mathbf{U}_{L}^{L} \nu_{L}, \quad e_{L}^{0}=\mathbf{U}_{L}^{L} \mathbf{V}_{e} e_{L}, \quad e_{R}^{0}=\mathbf{U}_{R}^{e} e_{R},
\end{aligned}
$$

where $\mathbf{U}_{L}^{Q, L}, \mathbf{U}_{R}^{u, d, e}$ are $3 \times 3$ unitary matrices and $\mathbf{V}_{d}$ and $\mathbf{V}_{e}^{\dagger}$ are the CKM and the PMNS mixing matrices, respectively. The individual components of the Higgs doublets $\Phi_{1,2}$ are given by

$$
\Phi_{1,2}=\left(\begin{array}{c}
\phi_{1,2}^{+} \\
\frac{1}{\sqrt{2}}\left(v_{1,2}+\phi_{1,2}+i a_{1,2}\right)
\end{array}\right) .
$$

In the CP-violating MSSM, the weak-state Higgs fields $\phi_{1,2}, a_{1,2}$ and $\phi_{1,2}^{-}$are related to the neutral CP-mixed mass eigenstates $H_{1,2,3}$, the charged Higgs boson $H^{-}$and the would-be Goldstone bosons $G^{0}$ and $G^{-}$, associated with the $Z$ and $W^{-}$bosons, through [38-47]:

$$
\begin{aligned}
\phi_{1} & =O_{1 i} H_{i}, & \phi_{2} & =O_{2 i} H_{i}, \\
a_{1} & =c_{\beta} G^{0}-s_{\beta} O_{3 i} H_{i}, & a_{2} & =s_{\beta} G^{0}+c_{\beta} O_{3 i} H_{i}, \\
\phi_{1}^{-} & =c_{\beta} G^{-}-s_{\beta} H^{-}, & \phi_{2}^{-} & =s_{\beta} G^{-}+c_{\beta} H^{-},
\end{aligned}
$$


where $s_{\beta} \equiv \sin \beta, c_{\beta} \equiv \cos \beta$ and $O$ is an orthogonal $3 \times 3$ Higgs-boson-mixing matrix.

The functional $\mathbf{h}_{f}^{-1} \Delta \mathbf{h}_{f}$ with $f=d, e, u$ can be written as

$$
\begin{aligned}
\mathbf{h}_{d, e}^{-1} \Delta \mathbf{h}_{d, e} & =\left(0, \boldsymbol{\Delta}_{d, e}\right)+\sum_{i=1,2}\left(\boldsymbol{\Delta}_{d, e}^{\phi_{i}^{-}} \phi_{i}^{-}, \frac{\boldsymbol{\Delta}_{d, e}^{\phi_{i}}}{\sqrt{2}} \phi_{i}+\frac{\boldsymbol{\Delta}_{d, e}^{a_{i}}}{\sqrt{2} i} a_{i}\right), \\
\mathbf{h}_{u}^{-1} \Delta \mathbf{h}_{u} & =\left(0, \boldsymbol{\Delta}_{u}\right)+\sum_{i=1,2}\left(\boldsymbol{\Delta}_{u}^{\phi_{i}^{+}} \phi_{i}^{+}, \frac{\boldsymbol{\Delta}_{u}^{\phi_{i}}}{\sqrt{2}} \phi_{i}+\frac{\boldsymbol{\Delta}_{u}^{a_{i}}}{\sqrt{2} i} a_{i}\right) .
\end{aligned}
$$

The $3 \times 3$ matrices $\boldsymbol{\Delta}_{f}, \boldsymbol{\Delta}_{f}^{\phi_{i}^{ \pm}}, \boldsymbol{\Delta}_{f}^{\phi_{i}}$, and $\boldsymbol{\Delta}_{f}^{a_{i}}$ are given by

$$
\begin{aligned}
\boldsymbol{\Delta}_{f} & =\left\langle\mathbf{h}_{f}^{-1} \Delta \mathbf{h}_{f}\right\rangle, & \boldsymbol{\Delta}_{f}^{\phi_{i}} & =\sqrt{2}\left\langle\frac{\delta}{\delta \phi_{i}} \mathbf{h}_{f}^{-1} \Delta \mathbf{h}_{f}\right\rangle, \\
\boldsymbol{\Delta}_{f}^{a_{i}} & =i \sqrt{2}\left\langle\frac{\delta}{\delta a_{i}} \mathbf{h}_{f}^{-1} \Delta \mathbf{h}_{f}\right\rangle, & \boldsymbol{\Delta}_{f}^{\phi_{i}^{ \pm}} & =\left\langle\frac{\delta}{\delta \phi_{i}^{ \pm}} \mathbf{h}_{f}^{-1} \Delta \mathbf{h}_{f}\right\rangle,
\end{aligned}
$$

where $\langle\ldots\rangle$ indicates taking the VEV of the enclosed expression. With these, we may conveniently express the general flavour-changing (FC) effective Lagrangian for the interactions of the neutral and charged Higgs fields to the up- and down-type quarks $u, d$ and to the charged leptons in the following form:

$$
\begin{aligned}
\mathcal{L}_{\mathrm{FC}}=-\frac{g}{2 M_{W}}[ & H_{i} \bar{d}\left(\widehat{\mathbf{M}}_{d} \mathbf{g}_{H_{i} \bar{d} d}^{L} P_{L}+\mathbf{g}_{H_{i} \bar{d} d}^{R} \widehat{\mathbf{M}}_{d} P_{R}\right) d+G^{0} \bar{d}\left(\widehat{\mathbf{M}}_{d} i \gamma_{5}\right) d \\
& +H_{i} \bar{u}\left(\widehat{\mathbf{M}}_{u} \mathbf{g}_{H_{i} \bar{u} u}^{L} P_{L}+\mathbf{g}_{H_{i} \bar{u} u}^{R} \widehat{\mathbf{M}}_{u} P_{R}\right) u-G^{0} \bar{u}\left(\widehat{\mathbf{M}}_{u} i \gamma_{5}\right) u \\
& \left.+H_{i} \bar{e}\left(\widehat{\mathbf{M}}_{e} \mathbf{g}_{H_{i} \bar{e} e}^{L} P_{L}+\mathbf{g}_{H_{i} \bar{e} e}^{R} \widehat{\mathbf{M}}_{e} P_{R}\right) e+G^{0} \bar{e}\left(\widehat{\mathbf{M}}_{e} i \gamma_{5}\right) e\right] \\
-\frac{g}{\sqrt{2} M_{W}} & {\left[H^{-} \bar{d}\left(\widehat{\mathbf{M}}_{d} \mathbf{g}_{H^{-} \bar{d} u}^{L} P_{L}+\mathbf{g}_{H^{-} \bar{d} u}^{R} \widehat{\mathbf{M}}_{u} P_{R}\right) u+H^{-} \bar{e}\left(\widehat{\mathbf{M}}_{e} \mathbf{g}_{H^{-} \bar{e} \nu}^{L} P_{L}\right) \nu\right.} \\
& \left.+G^{-} \bar{d}\left(\widehat{\mathbf{M}}_{d} \mathbf{V}_{d}^{\dagger} P_{L}-\mathbf{V}_{d^{\dagger}}^{\dagger} \widehat{\mathbf{M}}_{u} P_{R}\right) u+G^{-} \bar{e}\left(\widehat{\mathbf{M}}_{e} \mathbf{V}_{e}^{\dagger} P_{L}\right) \nu+\text { H.c. }\right],
\end{aligned}
$$

where $\widehat{\mathbf{M}}_{u, d, e}$ are the physical diagonal mass matrices for the up- and down-type quarks and charged leptons, which are related to the Yukawa coupling matrices by

$$
\left(\mathbf{U}_{R}^{d, e}\right)^{\dagger} \mathbf{h}_{d, e} \mathbf{U}_{L}^{Q, L}=\frac{\sqrt{2}}{v_{1}} \widehat{\mathbf{M}}_{d, e} \mathbf{V}_{d, e}^{\dagger} \mathbf{R}_{d, e}^{-1}, \quad\left(\mathbf{U}_{R}^{u}\right)^{\dagger} \mathbf{h}_{u} \mathbf{U}_{L}^{Q}=\frac{\sqrt{2}}{v_{2}} \widehat{\mathbf{M}}_{u} \mathbf{R}_{u}^{-1},
$$

with

$$
\begin{gathered}
\mathbf{R}_{d, e}=\mathbf{1}+\frac{\sqrt{2}}{v_{1}}\left(\mathbf{U}_{L}^{Q, L}\right)^{\dagger} \boldsymbol{\Delta}_{d, e} \mathbf{U}_{L}^{Q, L} \\
\mathbf{R}_{u}=\mathbf{1}+\frac{\sqrt{2}}{v_{2}}\left(\mathbf{U}_{L}^{Q}\right)^{\dagger} \boldsymbol{\Delta}_{u} \mathbf{U}_{L}^{Q}
\end{gathered}
$$


The neutral Higgs couplings to the down-type quarks and charged leptons are given by

$$
\begin{aligned}
\mathbf{g}_{H_{i} \bar{f} f}^{L}= & \frac{O_{1 i}}{c_{\beta}} \mathbf{V}_{f}^{\dagger} \mathbf{R}_{f}^{-1}\left[\mathbf{1}+\left(\mathbf{U}_{L}^{F}\right)^{\dagger} \boldsymbol{\Delta}_{f}^{\phi_{1}} \mathbf{U}_{L}^{F}\right] \mathbf{V}_{f}+\frac{O_{2 i}}{c_{\beta}} \mathbf{V}_{f}^{\dagger} \mathbf{R}_{f}^{-1}\left(\mathbf{U}_{L}^{F}\right)^{\dagger} \boldsymbol{\Delta}_{f}^{\phi_{2}} \mathbf{U}_{L}^{F} \mathbf{V}_{f} \\
& +i O_{3 i} t_{\beta} \mathbf{V}_{f}^{\dagger} \mathbf{R}_{f}^{-1}\left[\mathbf{1}+\left(\mathbf{U}_{L}^{F}\right)^{\dagger} \boldsymbol{\Delta}_{f}^{a_{1}} \mathbf{U}_{L}^{F}-\frac{1}{t_{\beta}}\left(\mathbf{U}_{L}^{F}\right)^{\dagger} \boldsymbol{\Delta}_{f}^{a_{2}} \mathbf{U}_{L}^{F}\right] \mathbf{V}_{f} \\
\mathbf{g}_{H_{i} \bar{f} f}^{R}= & \left(\mathbf{g}_{H_{i} \bar{f} f}^{L}\right)^{\dagger}
\end{aligned}
$$

with $f=d, e, F=Q, L$ and $t_{\beta} \equiv \tan \beta$. The neutral Higgs couplings to the up-type quarks are given by

$$
\begin{aligned}
\mathbf{g}_{H_{i} \bar{u} u}^{L}= & \frac{O_{1 i}}{s_{\beta}} \mathbf{R}_{u}^{-1}\left(\mathbf{U}_{L}^{Q}\right)^{\dagger} \boldsymbol{\Delta}_{u}^{\phi_{1}} \mathbf{U}_{L}^{Q}+\frac{O_{2 i}}{s_{\beta}} \mathbf{R}_{u}^{-1}\left[\mathbf{1}+\left(\mathbf{U}_{L}^{Q}\right)^{\dagger} \boldsymbol{\Delta}_{u}^{\phi_{2}} \mathbf{U}_{L}^{Q}\right] \\
& +i O_{3 i} t_{\beta}^{-1} \mathbf{R}_{u}^{-1}\left[\mathbf{1}-\left(\mathbf{U}_{L}^{Q}\right)^{\dagger} \boldsymbol{\Delta}_{u}^{a_{2}} \mathbf{U}_{L}^{Q}+t_{\beta}\left(\mathbf{U}_{L}^{Q}\right)^{\dagger} \boldsymbol{\Delta}_{u}^{a_{1}} \mathbf{U}_{L}^{Q}\right] \\
\mathbf{g}_{H_{i} \bar{u} u}^{R}= & \left(\mathbf{g}_{H_{i} \bar{u} u}^{L}\right)^{\dagger} .
\end{aligned}
$$

Finally, the coupling to the charged Higgs boson are given by

$$
\begin{aligned}
\mathbf{g}_{H^{-} \bar{d} u}^{L}= & -t_{\beta} \mathbf{V}_{d}^{\dagger} \mathbf{R}_{d}^{-1}\left[\mathbf{1}+\left(\mathbf{U}_{L}^{Q}\right)^{\dagger} \boldsymbol{\Delta}_{d}^{\phi_{1}^{-}} \mathbf{U}_{L}^{Q}\right]+\mathbf{V}_{d}^{\dagger} \mathbf{R}_{d}^{-1}\left(\mathbf{U}_{L}^{Q}\right)^{\dagger} \boldsymbol{\Delta}_{d}^{\phi_{2}^{-}} \mathbf{U}_{L}^{Q}, \\
\mathbf{g}_{H^{-} \bar{d} u}^{R}= & -t_{\beta}^{-1} \mathbf{V}_{d}^{\dagger}\left[\mathbf{1}+\left(\mathbf{U}_{L}^{Q}\right)^{\dagger}\left(\boldsymbol{\Delta}_{u}^{\phi_{2}^{+}}\right)^{\dagger} \mathbf{U}_{L}^{Q}\right]\left(\mathbf{R}_{u}^{-1}\right)^{\dagger} \\
& +\mathbf{V}_{d}^{\dagger}\left(\mathbf{U}_{L}^{Q}\right)^{\dagger}\left(\boldsymbol{\Delta}_{u}^{\phi_{1}^{+}}\right)^{\dagger} \mathbf{U}_{L}^{Q}\left(\mathbf{R}_{u}^{-1}\right)^{\dagger} \\
\mathbf{g}_{H^{-} \bar{e} \nu}^{L}= & -t_{\beta} \mathbf{V}_{e}^{\dagger} \mathbf{R}_{e}^{-1}\left[\mathbf{1}+\left(\mathbf{U}_{L}^{L}\right)^{\dagger} \boldsymbol{\Delta}_{e}^{\phi_{1}^{-}} \mathbf{U}_{L}^{L}\right]+\mathbf{V}_{e}^{\dagger} \mathbf{R}_{e}^{-1}\left(\mathbf{U}_{L}^{L}\right)^{\dagger} \boldsymbol{\Delta}_{e}^{\phi_{2}^{-}} \mathbf{U}_{L}^{L}
\end{aligned}
$$

Note that, in order to fully specify the Higgs couplings, one needs to know $\boldsymbol{\Delta}_{f}, \boldsymbol{\Delta}_{f}^{\phi_{i}}, \boldsymbol{\Delta}_{f}^{a_{i}}$, and $\boldsymbol{\Delta}_{f}^{\phi_{i}^{ \pm}}$with $i=1,2$ and $f=d, u, e$, as well as the rotation matrices $\mathbf{U}_{L}^{Q, L}$ and $\mathbf{V}_{d, e}$. Hence, the analytic results presented here generalise those given in $[15,33]$ beyond the SHI approximation in an arbitrary flavour basis.

One may now exploit the properties of gauge- and flavour-covariance of the effective functional $\mathbf{h}_{f}^{-1} \Delta \mathbf{h}_{f}\left[\Phi_{1}, \Phi_{2}\right]$ with $f=e, d, u$ to obtain useful relations. Explicitly, they should have the forms:

$$
\begin{aligned}
\mathbf{h}_{e, d}^{-1} \boldsymbol{\Delta} \mathbf{h}_{e, d}\left[\Phi_{1}, \Phi_{2}\right] & =\Phi_{1}^{\dagger} \mathbf{F}_{e, d}+\Phi_{2}^{\dagger} \mathbf{G}_{e, d} \\
\mathbf{h}_{u}^{-1} \boldsymbol{\Delta} \mathbf{h}_{u}\left[\Phi_{1}, \Phi_{2}\right] & =\Phi_{1}^{T} \mathbf{F}_{u}+\Phi_{2}^{T} \mathbf{G}_{u}
\end{aligned}
$$

where $\mathbf{F}_{e, d, u}\left(\Phi_{1}^{\dagger} \Phi_{1}, \Phi_{2}^{\dagger} \Phi_{2}, \Phi_{1}^{\dagger} \Phi_{2}, \Phi_{2}^{\dagger} \Phi_{1}\right)$ and $\mathbf{G}_{e, d, u}\left(\Phi_{1}^{\dagger} \Phi_{1}, \Phi_{2}^{\dagger} \Phi_{2}, \Phi_{1}^{\dagger} \Phi_{2}, \Phi_{2}^{\dagger} \Phi_{1}\right)$ are calculable $3 \times 3$-dimensional functionals which in general transform as $\mathbf{h}_{e, d, u}^{\dagger} \mathbf{h}_{e, d, u}$ under the flavour rotations (2.3). In the SHI approximation, $\mathbf{F}$ and $\mathbf{G}$ are simply constant matrices, leading 
to the relations

$$
\begin{array}{ll}
\boldsymbol{\Delta}_{e, d}=\frac{v_{1}}{\sqrt{2}} \mathbf{F}_{e, d}^{0}+\frac{v_{2}}{\sqrt{2}} \mathbf{G}_{e, d}^{0}, & \boldsymbol{\Delta}_{u}=\frac{v_{1}}{\sqrt{2}} \mathbf{F}_{u}^{0}+\frac{v_{2}}{\sqrt{2}} \mathbf{G}_{u}^{0}, \\
\boldsymbol{\Delta}_{e, d}^{\phi_{1}}=\boldsymbol{\Delta}_{e, d}^{a_{1}}=\boldsymbol{\Delta}_{e, d}^{\phi_{1}^{-}}=\mathbf{F}_{e, d}^{0}, & \boldsymbol{\Delta}_{u}^{\phi_{1}}=-\boldsymbol{\Delta}_{u}^{a_{1}}=\boldsymbol{\Delta}_{u}^{\phi_{1}^{+}}=\mathbf{F}_{u}^{0}, \\
\boldsymbol{\Delta}_{e, d}^{\phi_{2}}=\boldsymbol{\Delta}_{e, d}^{a_{2}}=\boldsymbol{\Delta}_{e, d}^{\phi_{2}^{-}}=\mathbf{G}_{e, d}^{0}, & \boldsymbol{\Delta}_{u}^{\phi_{2}}=-\boldsymbol{\Delta}_{u}^{a_{2}}=\boldsymbol{\Delta}_{u}^{\phi_{2}^{+}}=\mathbf{G}_{u}^{0} .
\end{array}
$$

Including the RG-induced flavour-violating terms, the constant matrices $\mathbf{F}$ and $\mathbf{G}$ take on the form:

$$
\mathbf{F}_{e, d, u}^{0}=\left\langle\boldsymbol{\Delta}_{e, d, u}^{\Phi_{1}}+\boldsymbol{\delta} \boldsymbol{\Delta}_{e, d, u}^{\Phi_{1}}\right\rangle_{0}, \quad \mathbf{G}_{e, d, u}^{0}=\left\langle\boldsymbol{\Delta}_{e, d, u}^{\Phi_{2}}+\boldsymbol{\delta} \boldsymbol{\Delta}_{e, d, u}^{\Phi_{2}}\right\rangle_{0},
$$

where the flavour-non-universal terms are given by the set of equations (3.11), (3.12), (3.24), (3.25), (3.31) and (3.32). In the next section, we will present numerical estimates in the SHI approximation, including the RG-induced flavour-non-universal contributions to the threshold corrections.

\subsection{RG effects on FCNC Higgs-Boson couplings}

We may now illustrate the significance of the results presented in the previous section, by analyzing the variations of the FCNC couplings of the neutral Higgs bosons to down-type quarks as functions of $\tan \beta$ in the same MCPMFV scenario (2.17) introduced previously. ${ }^{4}$

To start with, we display in figure 10 the variations with $\tan \beta$ of the couplings $\left|g^{L}\left(H_{1,2,3} \bar{s} d\right)\right|$ (top row), $\left|g^{L}\left(H_{1,2,3} \bar{b} d\right)\right|$ (middle row) and $\left|g^{L}\left(H_{1,2,3} \bar{b} s\right)\right|$, for various values of $\Phi_{M}$ as given in figure 1. We see that, for each of the Higgs particles, we have the expected hierarchy across fermion generations, i.e., $\left|g^{L}\left(H_{1,2,3} \bar{s} d\right)\right| /\left|g^{L}\left(H_{1,2,3} \bar{b} d\right)\right| \sim\left|\left(\mathbf{V}_{d}\right)_{t s}\right| \sim 0.04$ and $\left|g^{L}\left(H_{1,2,3} \bar{b} d\right)\right| /\left|g^{L}\left(H_{1,2,3} \bar{b} s\right)\right| \sim\left|\left(\mathbf{V}_{d}\right)_{t d} /\left(\mathbf{V}_{d}\right)_{t s}\right| \sim 0.2$. Again, we observe that for $\Phi_{M}=180^{\circ}$, gluino threshold effects increase the value of the threshold matrix $\mathbf{R}_{d}^{-1}$, thus giving rise to larger FCNC Higgs-boson couplings.

In the top left panel of figure 11, we show the dependence of the couplings $\left|g^{L}\left(H_{1,2,3} \bar{b} s\right)\right|$ on $\tan \beta$ for $\Phi_{M}=0$, whilst the remaining panels show the correlations between this coupling and each of the expansion coefficients $\widetilde{m}_{Q}^{2, I} / \widetilde{m}_{Q}^{2,0}$ for the same values of $\tan \beta$. As expected from the results displayed in figure 1 , the variation in the coefficients is extremely small.

Finally, in figure 12 we illustrate the impact of the individual flavour-violating components induced by RG running on the FCNC Higgs-boson couplings $\left|g^{L}\left(H_{1,2,3} \bar{b} s\right)\right|$. Specifically, we plot the couplings $\left|g^{L}\left(H_{1,2,3} \bar{b} s\right)\right|$ against the "level" $L$, defined by limiting the sums

$$
\begin{aligned}
\widetilde{\mathbf{M}}_{Q, U, D}^{2(L)} & =\sum_{I=0}^{L} \widetilde{m}_{Q, U, D}^{2, I} \mathbf{H}_{I}^{Q, U, D} \\
\mathbf{a}_{u, d}^{(L)} & =\sum_{I=0}^{L} a_{u, d}^{I} \mathbf{h}_{u, d} \mathbf{H}_{I}^{Q}
\end{aligned}
$$

\footnotetext{
${ }^{4}$ We recall that, in this scenario, the heavier neutral Higgs bosons $H_{2,3}$ are almost degenerate, whereas $H_{1}$ is significantly lighter.
} 

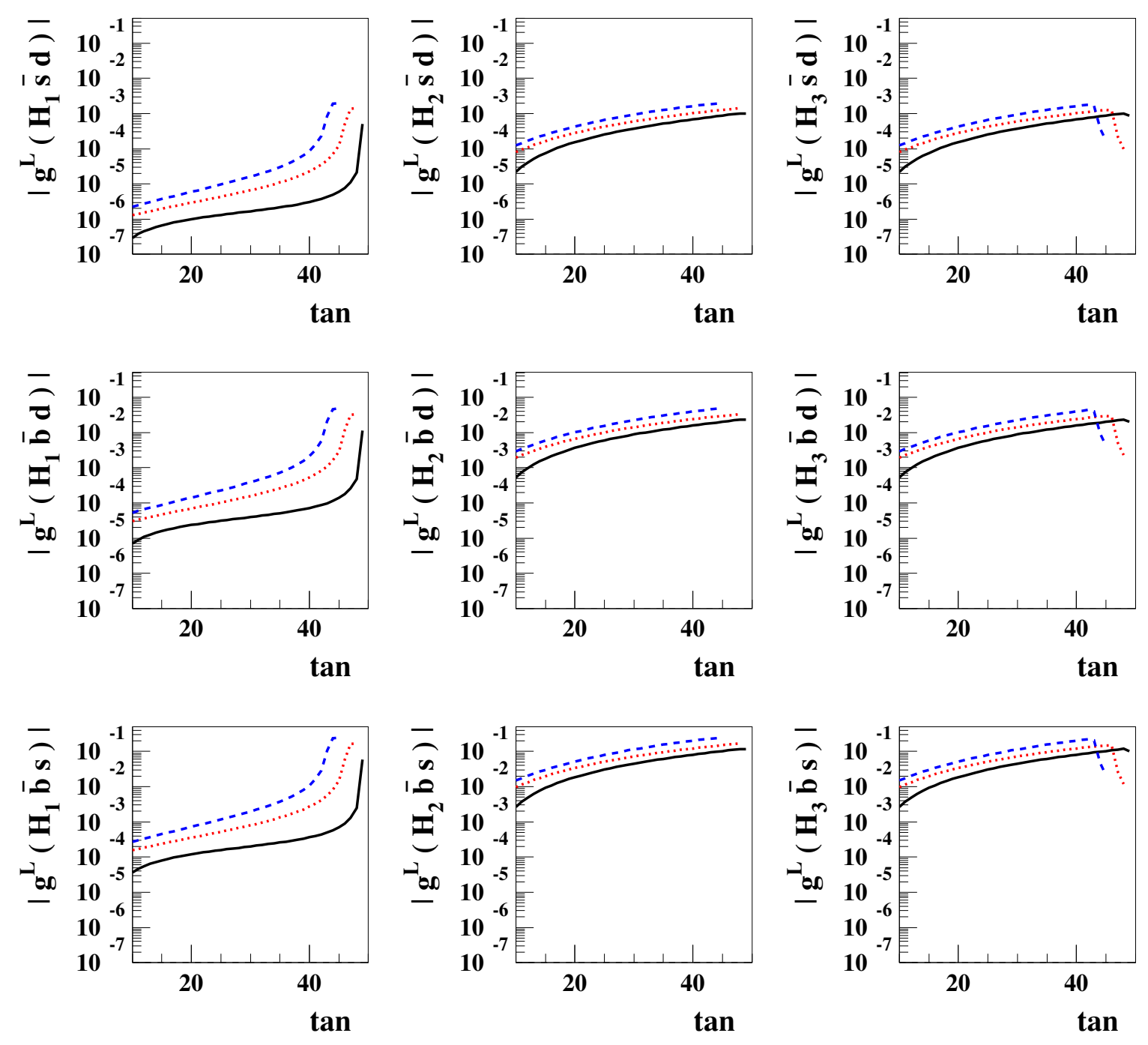

Figure 10. In the upper, middle, and lower panels we display the variation with $\tan \beta$ of the flavour-non-diagonal couplings $\left|g_{H_{i} \bar{s} d}^{L}\right|,\left|g_{H_{i} \bar{b} d}^{L}\right|$, and $\left|g_{H_{i} \bar{b} s}^{L}\right|$, respectively, of the neutral Higgs bosons $H_{1}$ (left column), $H_{2}$ (middle column), and $H_{3}$ (right column). In each panel, the lines are the same as in figure 1. The input MCPMFV SUSY-breaking parameters are taken to be the same as in $(2.17)$.

The level $L=0$ indicates that the couplings are calculated including only the first coefficient in the expansion of the soft matrices, i.e. the flavour-singlet component $\propto \mathbf{1}_{3}$. On the other hand, the highest level $L=8$ gives the complete reconstruction of the soft SUSY-breaking matrices in a flavour-basis expansion, i.e. $\widetilde{\mathbf{M}}_{Q, U, D}^{2(8)} \equiv \widetilde{\mathbf{M}}_{Q, U, D}^{2}$ and $\mathbf{a}_{u, d}^{(8)} \equiv \mathbf{a}_{u, d}$.

We also display in figure 12 numerical estimates for various values of $\tan \beta$ and $\Phi_{M}$. 

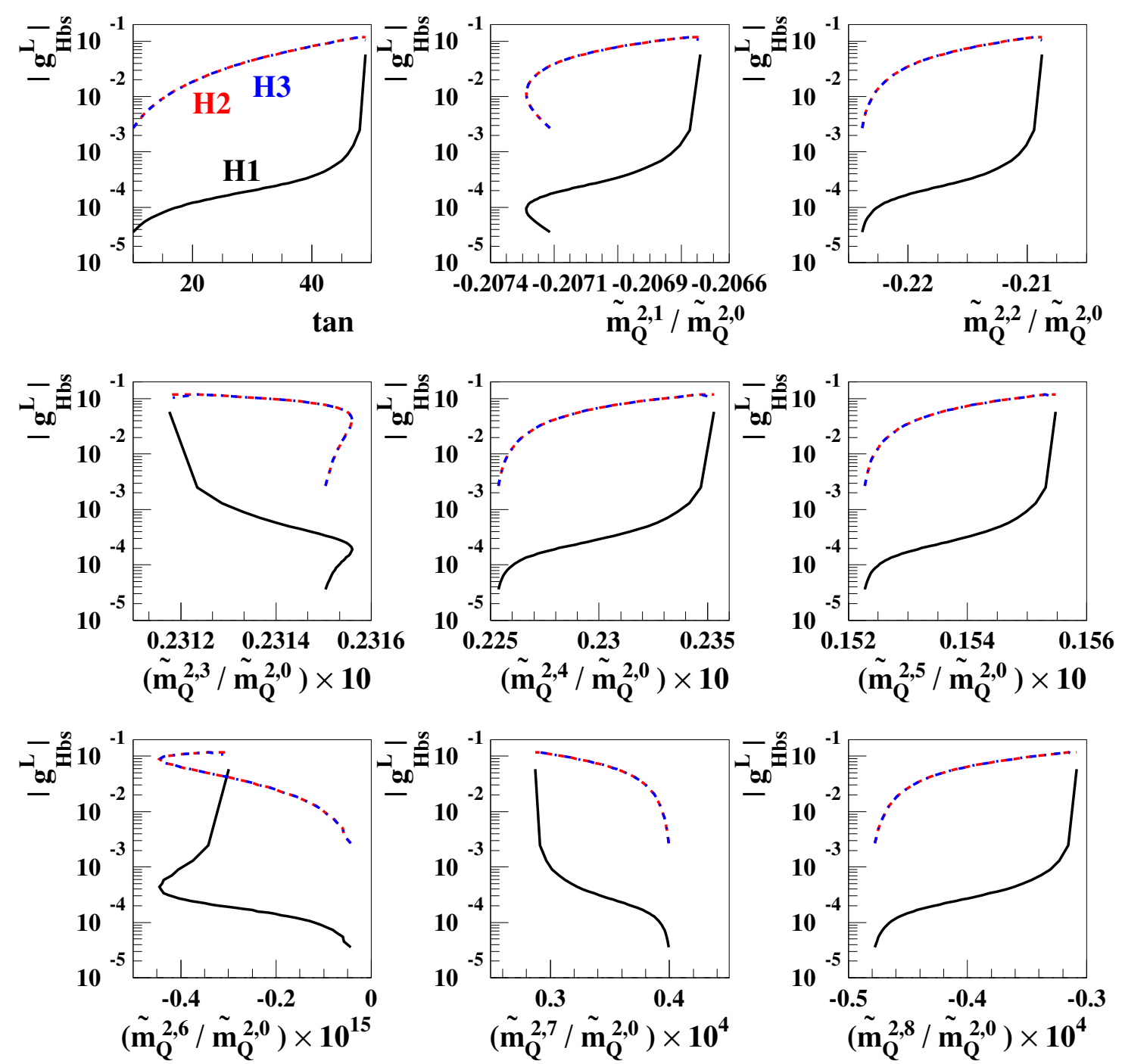

Figure 11. In the top left panel we display the variation with $\tan \beta$ of the flavour-non-diagonal couplings $\left|g_{H_{i} \bar{b} s}^{L}\right|$ of the neutral Higgs bosons $H_{1,2,3}$ taking $\Phi_{M}=0^{\circ}$. In the other panels we display the correlations of these couplings with the ratios $\widetilde{m}_{Q}^{2, I} / \widetilde{m}_{Q}^{2,0}$ for the same value of $\Phi_{M}=0^{\circ}$. The input MCPMFV SUSY-breaking parameters are taken to be the same as in (2.17).

In all different panels, we observe that the effective Higgs-boson couplings remain flavourviolating, even if the soft SUSY-breaking matrices are flavour-singlet. In this case, the Higgsino-mediated threshold correction and the 2HDM contribution, both of which are $\propto \mathbf{h}_{u}^{\dagger} \mathbf{h}_{u}$, remain the dominant source of flavour violation when all the soft SUSY-breaking matrices are proportional to $\mathbf{1}_{3}$. Interestingly enough, the RG-generated flavour-changing terms in the soft matrices are found to produce a cancellation against the leading level 

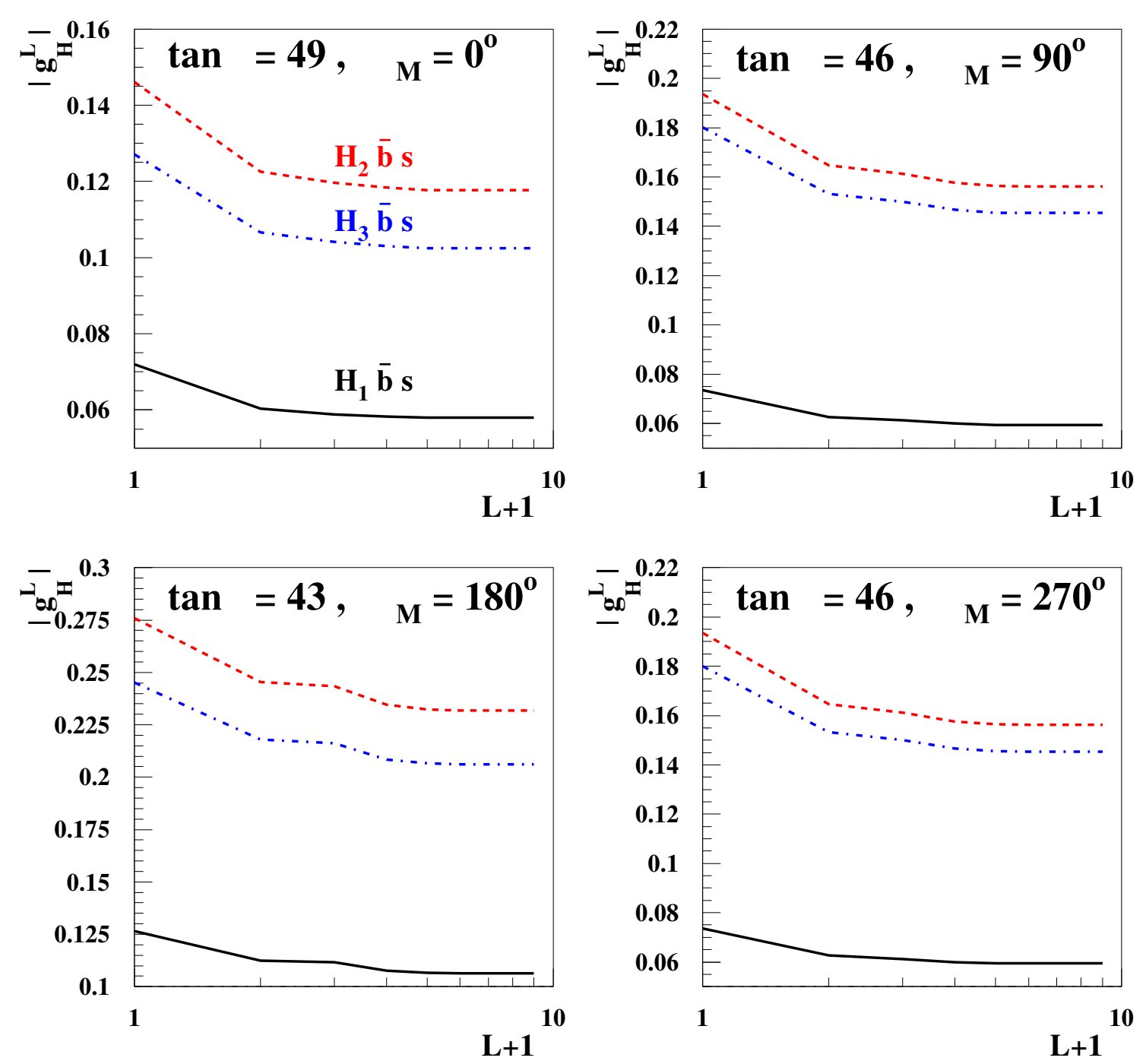

Figure 12. In the top left panel we display the variation with the level $L$ of the flavour-nondiagonal couplings $\left|g_{H_{i} \bar{b} s}^{L}\right|$ of the neutral Higgs bosons $H_{1,2,3}$ taking $\tan \beta=49$ and $\Phi_{M}=0^{\circ}$. The level $L$ is defined in the sum: $\widetilde{\mathbf{M}}_{Q, U, D}^{2}=\sum_{I=0}^{L} \widetilde{m}_{Q, U, D}^{2, I} \mathbf{H}_{I}^{Q, U, D}$ and $\mathbf{a}_{u, d}=\sum_{I=0}^{L} a_{u, d}^{I} \mathbf{h}_{u, d} \mathbf{H}_{I}^{Q}$. For example, $L=0$ implies that the couplings $\left|g_{H_{i} \bar{b} s}^{L}\right|$ are calculated only including the zero-th term in the expansions of the matrices $\widetilde{\mathbf{M}}_{Q, U, D}^{2}$ and $\mathbf{a}_{u, d}$ while $L=8$ means including all 9 terms in the expansions. In the other panels we display the variations for other values of $\tan \beta$ and $\Phi_{M}$ as shown. The input MCPMFV SUSY-breaking parameters are taken to be the same as in (2.17).

$L=0$ terms. This screening phenomenon is typically at the level of $\sim 20 \%$ of the Higgsino contribution. The largest non-flavour-singlet contribution is due to the level $L=1$ coefficients, although the $L=2,3$ coefficients also lead to appreciable effects for some 
values of $\Phi_{M}$. Thus, our analysis shows that the non-singlet flavour components within MCPMFV-type scenarios can modify the strength of the FCNC Higgs-boson couplings in a phenomenologically relevant way.

\section{Conclusions}

We have presented a new geometric method for the flavour decomposition of a general soft SUSY-breaking sector in the MSSM. We have shown how the up- and down-type quark Yukawa-coupling matrices may be used to furnish a complete geometric basis for the flavour space of the soft SUSY-breaking squark mass matrices and trilinear couplings. Such a decomposition in the leptonic sector would require the extension of the MSSM by right-handed neutrinos and the introduction of neutrino Yukawa couplings.

The MFV decomposition developed here is valid except possibly in the limit where the effective Yukawa couplings are rendered real after the inclusion of threshold corrections. In this case, an alternative basis must be chosen; the tree-level quark Yukawa couplings are a suitable choice. Conversely, if the tree-level Yukawa couplings are real in a MSSM scenario with soft $\mathrm{CP}$ violation, then RG effects alone cannot alter this fact. In such a scenario, the effective Yukawa couplings are expected to acquire a non-trivial KM phase induced by the complex soft SUSY-breaking parameters and they will thus provide a complete basis for flavour decomposition.

It is obvious that threshold corrections to the couplings of the MSSM Higgs bosons to fermions play a central role to the construction of a non-degenerate flavour geometry as developed here. In this context, we have presented the complete one-loop threshold corrections, showing explicitly how non-trivial flavour geometry of the soft SUSY-breaking matrices can become an additional source for FCNC effects on the Higgs-boson couplings to fermions. We have then used the flavour-space decomposition to examine the behaviour of these couplings. We have observed that flavour violation due to the RG evolution of the soft SUSY-breaking matrices from the GUT to the electroweak scale leads to a screening phenomenon of order $\sim 20 \%$ for the effective Higgs-fermion vertices, which could be phenomenologically relevant.

In this paper we have applied our flavour geometric approach to one process, namely to the Higgs-boson FCNC couplings to fermions. Non-trivial flavour structures amongst the soft SUSY-breaking matrices are known to contribute to a number of processes at the one-loop level, such as $b \rightarrow s \gamma$ decays and neutral meson mixing [48]. Applying the framework developed here to these processes would lead to constraints on the magnitudes of the various coefficients which would in turn have implications for the validity of the Minimal Flavour Violation hypothesis.

\section{Acknowledgments}

The work of AP was supported in part by the STFC research grant: PP/D000157/1. RNH acknowledges support from the Japan Society for the Promotion of Science. 


\section{A Background Higgs-Field-Dependent mass matrices}

Here we collect explicit tree-level forms for all the mass matrices which appear in the calculations of section 3 .

\section{A.1 Squark mass-squared matrix}

The $12 \times 12$ squark mass-squared matrix $\widetilde{\mathbf{M}}^{2}$ may be expressed as [15]

$$
\widetilde{\mathbf{M}}^{2}\left[\Phi_{1}, \Phi_{2}, S\right]=\left(\begin{array}{ll}
\left(\widetilde{\mathbf{M}}^{2}\right)_{\tilde{Q}^{\dagger} \tilde{Q}} & \left(\widetilde{\mathbf{M}}^{2}\right)_{\tilde{Q}^{\dagger} \tilde{U}}\left(\widetilde{\mathbf{M}}^{2}\right)_{\tilde{Q}^{\dagger} \tilde{D}} \\
\left(\widetilde{\mathbf{M}}^{2}\right)_{\tilde{U}^{\dagger} \tilde{Q}}\left(\widetilde{\mathbf{M}}^{2}\right)_{\tilde{U}^{\dagger} \tilde{U}}\left(\widetilde{\mathbf{M}}^{2}\right)_{\tilde{U}^{\dagger} \tilde{D}} \\
\left(\widetilde{\mathbf{M}}^{2}\right)_{\tilde{D}^{\dagger} \tilde{Q}}\left(\widetilde{\mathbf{M}}^{2}\right)_{\tilde{D}^{\dagger} \tilde{U}}\left(\widetilde{\mathbf{M}}^{2}\right)_{\tilde{D}^{\dagger} \tilde{D}}
\end{array}\right)_{i j}
$$

with

$$
\begin{aligned}
\left(\widetilde{\mathbf{M}}^{2}\right)_{\tilde{Q}_{i}^{\dagger} \tilde{Q}_{j}}= & \left(\widetilde{\mathbf{M}}_{Q}^{2}\right)_{i j} \mathbf{1}_{2}+\left(\mathbf{h}_{d}^{\dagger} \mathbf{h}_{d}\right)_{i j} \Phi_{1} \Phi_{1}^{\dagger}+\left(\mathbf{h}_{u}^{\dagger} \mathbf{h}_{u}\right)_{i j}\left(\Phi_{2}^{\dagger} \Phi_{2} \mathbf{1}_{2}-\Phi_{2} \Phi_{2}^{\dagger}\right) \\
& -\frac{1}{2} \delta_{i j} g_{2}^{2}\left(\Phi_{1} \Phi_{1}^{\dagger}-\Phi_{2} \Phi_{2}^{\dagger}\right)+\delta_{i j}\left(\frac{1}{4} g_{2}^{2}-\frac{1}{12} g_{1}^{2}\right)\left(\Phi_{1}^{\dagger} \Phi_{1}-\Phi_{2}^{\dagger} \Phi_{2}\right) \mathbf{1}_{2} \\
\left(\widetilde{\mathbf{M}}^{2}\right)_{\tilde{U}_{i}^{\dagger} \tilde{Q}_{j}}= & \left(\widetilde{\mathbf{M}}^{2}\right)_{\tilde{Q}_{j}^{\dagger} \tilde{U}_{i}}^{\dagger}=-\left(\mathbf{a}_{u}\right)_{i j} \Phi_{2}^{T} i \sigma_{2}+\left(\mathbf{h}_{u}\right)_{i j} \mu^{*} \Phi_{1}^{T} i \sigma_{2}, \\
\left(\widetilde{\mathbf{M}}^{2}\right)_{\tilde{D}_{i}^{\dagger} \tilde{Q}_{j}}= & \left(\widetilde{\mathbf{M}}^{2}\right)_{\tilde{Q}_{j}^{\dagger} \tilde{D}_{i}}^{\dagger}=+\left(\mathbf{a}_{d}\right)_{i j} \Phi_{1}^{\dagger}-\left(\mathbf{h}_{d}\right)_{i j} \mu^{*} \Phi_{2}^{\dagger} \\
\left(\widetilde{\mathbf{M}}^{2}\right)_{\tilde{U}_{i}^{\dagger} \tilde{U}_{j}}= & \left(\widetilde{\mathbf{M}}_{U}^{2}\right)_{i j}+\left(\mathbf{h}_{u} \mathbf{h}_{u}^{\dagger}\right)_{i j} \Phi_{2}^{\dagger} \Phi_{2}+\frac{1}{3} \delta_{i j} g_{1}^{2}\left(\Phi_{1}^{\dagger} \Phi_{1}-\Phi_{2}^{\dagger} \Phi_{2}\right) \\
\left(\widetilde{\mathbf{M}}^{2}\right)_{\tilde{D}_{i}^{\dagger} \tilde{D}_{j}}= & \left(\widetilde{\mathbf{M}}_{D}^{2}\right)_{i j}+\left(\mathbf{h}_{d} \mathbf{h}_{d}^{\dagger}\right)_{i j} \Phi_{1}^{\dagger} \Phi_{1}-\frac{1}{6} \delta_{i j} g_{1}^{2}\left(\Phi_{1}^{\dagger} \Phi_{1}-\Phi_{2}^{\dagger} \Phi_{2}\right) \\
\left(\widetilde{\mathbf{M}}^{2}\right)_{\tilde{U}_{i}^{\dagger} \tilde{D}_{j}}= & \left(\widetilde{\mathbf{M}}^{2}\right)_{\tilde{D}_{j}^{\dagger} \tilde{U}_{i}}^{\dagger}=\left(\mathbf{h}_{u} \mathbf{h}_{d}^{\dagger}\right)_{i j} \Phi_{1}^{T} i \sigma \Phi_{2}
\end{aligned}
$$

where $\widetilde{\mathbf{M}}_{Q}^{2}$, $\widetilde{\mathbf{M}}_{U}^{2}$ and $\widetilde{\mathbf{M}}_{D}^{2}$ are the soft SUSY-breaking squark mass matrices and $\mu$ is the coefficient of the Higgs bilinear term of the MSSM superpotential.

\section{A.2 Chargino-Neutralino mass matrix}

The $8 \times 8$ chargino-neutralino mass matrix $\mathbf{M}_{C}$ which appears in (3.4) is given by

$$
\mathbf{M}_{C}=\left(\begin{array}{cccc}
M_{1} & 0 & \frac{1}{\sqrt{2}} g_{1} \Phi_{2}^{\dagger} & \frac{1}{\sqrt{2}} g_{1} \Phi_{1}^{T}\left(i \sigma_{2}\right) \\
0 & M_{2} \mathbf{1}_{3} & \frac{1}{\sqrt{2}} g_{2} \Phi_{2}^{\dagger} \sigma_{i} & -\frac{1}{\sqrt{2}} g_{2} \Phi_{1}^{T}\left(i \sigma_{2}\right) \sigma_{i} \\
\frac{1}{\sqrt{2}} g_{1} \Phi_{2}^{*} & \frac{1}{\sqrt{2}} g_{2} \sigma_{i}^{T} \Phi_{2}^{*} & \mathbf{0}_{2} & \mu\left(i \sigma_{2}\right) \\
-\frac{1}{\sqrt{2}}\left(i \sigma_{2}\right) g_{1} \Phi_{1} & \frac{1}{\sqrt{2}} g_{2} \sigma_{i}^{T}\left(i \sigma_{2}\right) \Phi_{1} & -\mu\left(i \sigma_{2}\right) & \mathbf{0}_{2}
\end{array}\right) .
$$

\section{A.3 Slepton mass-squared matrix}

The $9 \times 9$ slepton mass-squared matrix $\widetilde{\mathbf{M}}_{l}^{2}$ may be expressed as

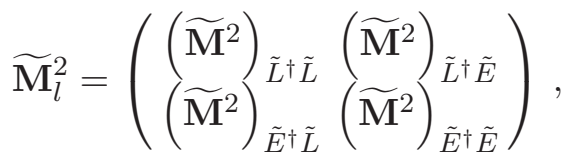


with

$$
\begin{aligned}
\left(\widetilde{\mathbf{M}}_{l}^{2}\right)_{\tilde{L}^{\dagger} \tilde{L}}= & \left(\widetilde{\mathbf{M}}_{L}^{2}\right)_{i j} \mathbf{1}_{2}+\left(\mathbf{h}_{e}^{\dagger} \mathbf{h}_{e}\right)_{i j} \Phi_{1} \Phi_{1}^{\dagger}-\frac{1}{2} \delta_{i j} g_{2}^{2}\left(\Phi_{1} \Phi_{1}^{\dagger}-\Phi_{2} \Phi_{2}^{\dagger}\right) \\
& +\frac{1}{4} \delta_{i j}\left(g_{2}^{2}+g_{1}^{2}\right)\left(\Phi_{1}^{\dagger} \Phi_{1}-\Phi_{2}^{\dagger} \Phi_{2}\right) \mathbf{1}_{2} \\
\left(\widetilde{\mathbf{M}}_{l}^{2}\right)_{\tilde{E} \dagger \tilde{L}}= & \left(\widetilde{\mathbf{M}}_{l}^{2}\right)_{\tilde{L}^{\dagger} \tilde{E}}^{\dagger}=+\left(\mathbf{a}_{e}\right)_{i j} \Phi_{1}^{\dagger}-\left(\mathbf{h}_{e}\right)_{i j} \mu^{*} \Phi_{2}^{\dagger}, \\
\left(\widetilde{\mathbf{M}}_{l}^{2}\right)_{\tilde{E}^{\dagger} \tilde{E}}= & \left(\widetilde{\mathbf{M}}_{E}^{2}\right)_{i j}+\left(\mathbf{h}_{e} \mathbf{h}_{e}^{\dagger}\right)_{i j} \Phi_{1}^{\dagger} \Phi_{1}-\frac{1}{2} \delta_{i j} g_{1}{ }^{2}\left(\Phi_{1}^{\dagger} \Phi_{1}-\Phi_{2}^{\dagger} \Phi_{2}\right) .
\end{aligned}
$$

Here $\widetilde{\mathbf{M}}_{L}^{2}$ and $\widetilde{\mathbf{M}}_{E}^{2}$ are the soft SUSY-breaking slepton mass matrices.

\section{A.4 Higgs-boson mass-squared matrix}

The $8 \times 8$ Higgs-boson mass-squared matrix $\mathbf{M}_{H}^{2}$ is given at tree level in the basis $\left(\Phi_{1}, \Phi_{2}, \Phi_{1}^{*}, \Phi_{2}^{*}\right)^{T}$ by

$$
\mathbf{M}_{H}^{2}\left[\Phi_{1}, \Phi_{2}\right]=\left(\begin{array}{cccc}
\left(\mathbf{M}_{H}^{2}\right)_{\Phi_{1}^{\dagger} \Phi_{1}} & \left(\mathbf{M}_{H}^{2}\right)_{\Phi_{1}^{\dagger} \Phi_{2}} & \left(\mathbf{M}_{H}^{2}\right)_{\Phi_{1}^{\dagger} \Phi_{1}^{*}} & \left(\mathbf{M}_{H}^{2}\right)_{\Phi_{1}^{\dagger} \Phi_{2}^{*}} \\
\left(\mathbf{M}_{H}^{2}\right)_{\Phi_{2}^{\dagger} \Phi_{1}} & \left(\mathbf{M}_{H}^{2}\right)_{\Phi_{2}^{\dagger} \Phi_{2}} & \left(\mathbf{M}_{H}^{2}\right)_{\Phi_{2}^{\dagger} \Phi_{1}^{*}} & \left(\mathbf{M}_{H}^{2}\right)_{\Phi_{2}^{\dagger} \Phi_{2}^{*}} \\
\left(\mathbf{M}_{H}^{2}\right)_{\Phi_{1}^{T} \Phi_{1}} & \left(\mathbf{M}_{H}^{2}\right)_{\Phi_{1}^{T} \Phi_{2}} & \left(\mathbf{M}_{H}^{2}\right)_{\Phi_{1}^{T} \Phi_{1}^{*}} & \left(\mathbf{M}_{H}^{2}\right)_{\Phi_{1}^{T} \Phi_{2}^{*}} \\
\left(\mathbf{M}_{H}^{2}\right)_{\Phi_{2}^{T} \Phi_{1}} & \left(\mathbf{M}_{H}^{2}\right)_{\Phi_{2}^{T} \Phi_{2}} & \left(\mathbf{M}_{H}^{2}\right)_{\Phi_{2}^{T} \Phi_{1}^{*}} & \left(\mathbf{M}_{H}^{2}\right)_{\Phi_{2}^{T} \Phi_{2}^{*}}
\end{array}\right)
$$

where

$$
\begin{aligned}
\left(\mathbf{M}_{H}^{2}\right)_{\Phi_{1}^{\dagger} \Phi_{1}} & =\left(\mathbf{M}_{H}^{2}\right)_{\Phi_{1}^{T} \Phi_{1}^{*}}^{T} \\
& =\left(M_{H_{d}}^{2}+|\mu|^{2}+\frac{g_{1}^{2}+g_{2}^{2}}{2} \Phi_{1}^{\dagger} \Phi_{1}+\frac{g_{2}^{2}-g_{1}^{2}}{4} \Phi_{2}^{\dagger} \Phi_{2}\right) \mathbf{1}_{2}-\frac{g_{2}^{2}}{2} \Phi_{2} \Phi_{2}^{\dagger}, \\
\left(\mathbf{M}_{H}^{2}\right)_{\Phi_{2}^{\dagger} \Phi_{2}} & =\left(\mathbf{M}_{H}^{2}\right)_{\Phi_{2}^{T} \Phi_{2}^{*}}^{T} \\
& =\left(M_{H_{u}}^{2}+|\mu|^{2}+\frac{g_{1}^{2}+g_{2}^{2}}{2} \Phi_{2}^{\dagger} \Phi_{2}+\frac{g_{2}^{2}-g_{1}^{2}}{4} \Phi_{1}^{\dagger} \Phi_{1}\right) \mathbf{1}_{2}-\frac{g_{2}^{2}}{2} \Phi_{1} \Phi_{1}^{\dagger}, \\
\left(\mathbf{M}_{H}^{2}\right)_{\Phi_{1}^{T} \Phi_{2}} & =\left(\mathbf{M}_{H}^{2}\right)_{\Phi_{2}^{T} \Phi_{1}}^{T}=\left(\mathbf{M}_{H}^{2}\right)_{\Phi_{2}^{\dagger} \Phi_{1}^{*}}^{\dagger}=\left(\mathbf{M}_{H}^{2}\right)_{\Phi_{1}^{\dagger} \Phi_{2}^{*}}^{*} \\
& =\frac{g_{2}^{2}-g_{1}^{2}}{4} \Phi_{1}^{*} \Phi_{2}^{\dagger}-\frac{g_{2}^{2}}{2} \Phi_{2}^{*} \Phi_{1}^{\dagger}, \\
\left(\mathbf{M}_{H}^{2}\right)_{\Phi_{1}^{\dagger} \Phi_{2}} & =\left(\mathbf{M}_{H}^{2}\right)_{\Phi_{2}^{\dagger} \Phi_{1}}^{\dagger}=\left(\mathbf{M}_{H}^{2}\right)_{\Phi_{2}^{T} \Phi_{1}}^{T}=\left(\mathbf{M}_{H}^{2}\right)_{\Phi_{1}^{T} \Phi_{2}^{*}}^{*} \\
& =-\left(B \mu+\frac{g_{2}^{2}}{2} \Phi_{2}^{\dagger} \Phi_{1}\right) \mathbf{1}_{2}+\frac{g_{2}^{2}-g_{1}^{2}}{4} \Phi_{1} \Phi_{2}^{\dagger}, \\
\left(\mathbf{M}_{H}^{2}\right)_{\Phi_{1}^{T} \Phi_{1}} & =\left(\mathbf{M}_{H}^{2}\right)_{\Phi_{1}^{\dagger} \Phi_{1}^{*}}^{*}=\frac{g_{2}^{2}+g_{1}^{2}}{2} \Phi_{1}^{*} \Phi_{1}^{\dagger}, \\
\left(\mathbf{M}_{H}^{2}\right)_{\Phi_{2}^{T} \Phi_{2}} & =\left(\mathbf{M}_{H}^{2}\right)_{\Phi_{2}^{\dagger} \Phi_{2}^{*}}^{*}=\frac{g_{2}^{2}+g_{1}^{2}}{2} \Phi_{2}^{*} \Phi_{2}^{\dagger} .
\end{aligned}
$$

\section{A.5 Quark mass matrix}

The $6 \times 6$ quark mass matrix $\mathbf{M}_{q}$ is given by

$$
\mathbf{M}_{q}\left[\Phi_{1}, \Phi_{2}\right]=\left(\begin{array}{c}
\left(\mathbf{M}_{q}\right)_{u_{i} Q_{j}} \\
\left(\mathbf{M}_{q}\right)_{d_{i} Q_{j}}
\end{array}\right)=\left(\begin{array}{c}
\left(\mathbf{h}_{u}\right)_{i j} \Phi_{2}^{T}\left(-i \sigma_{2}\right) \\
\left(\mathbf{h}_{d}\right)_{i j} \Phi_{1}^{\dagger}
\end{array}\right) .
$$


Open Access. This article is distributed under the terms of the Creative Commons Attribution Noncommercial License which permits any noncommercial use, distribution, and reproduction in any medium, provided the original author(s) and source are credited.

\section{References}

[1] H.P. Nilles, Supersymmetry, supergravity and particle physics, Phys. Rept. 110 (1984) 1 [SPIRES].

[2] H.E. Haber and G.L. Kane, The search for supersymmetry: probing physics beyond the Standard Model, Phys. Rept. 117 (1985) 75 [SPIRES].

[3] D.J.H. Chung et al., The soft supersymmetry-breaking Lagrangian: theory and applications, Phys. Rept. 407 (2005) 1 [hep-ph/0312378] [SPIRES].

[4] T. Ibrahim and P. Nath, CP violation from standard model to strings, Rev. Mod. Phys. 80 (2008) 577 [arXiv: 0705. 2008] [SPIRES].

[5] N. Cabibbo, Unitary symmetry and leptonic decays, Phys. Rev. Lett. 10 (1963) 531 [SPIRES].

[6] M. Kobayashi and T. Maskawa, CP violation in the renormalizable theory of weak interaction, Prog. Theor. Phys. 49 (1973) 652 [SPIRES].

[7] S.L. Glashow, J. Iliopoulos and L. Maiani, Weak interactions with Lepton-Hadron symmetry, Phys. Rev. D 2 (1970) 1285 [SPIRES].

[8] R.S. Chivukula and H. Georgi, Composite technicolor Standard Model, Phys. Lett. B 188 (1987) 99 [SPIRES].

[9] G. D'Ambrosio, G.F. Giudice, G. Isidori and A. Strumia, Minimal flavour violation: An effective field theory approach, Nucl. Phys. B 645 (2002) 155 [hep-ph/0207036] [SPIRES].

[10] G.G. Ross, L. Velasco-Sevilla and O. Vives, Spontaneous CP-violation and non-Abelian family symmetry in SUSY, Nucl. Phys. B 692 (2004) 50 [hep-ph/0401064] [SPIRES].

[11] S. Antusch, S.F. King and M. Malinsky, Solving the SUSY flavour and CP problems with SU(3) family symmetry, JHEP 06 (2008) 068 [arXiv:0708.1282] [SPIRES].

[12] P. Paradisi, M. Ratz, R. Schieren and C. Simonetto, Running minimal flavor violation, Phys. Lett. B 668 (2008) 202 [arXiv:0805.3989] [SPIRES].

[13] G. Colangelo, E. Nikolidakis and C. Smith, Supersymmetric models with minimal flavour violation and their running, Eur. Phys. J. C 59 (2009) 75 [arXiv:0807.0801] [SPIRES].

[14] L. Mercolli and C. Smith, EDM constraints on flavored CP-violating phases, Nucl. Phys. B 817 (2009) 1 [arXiv:0902.1949] [SPIRES].

[15] J.R. Ellis, J.S. Lee and A. Pilaftsis, B-Meson observables in the maximally CP-violating MSSM with minimal flavour violation, Phys. Rev. D 76 (2007) 115011 [arXiv:0708.2079] [SPIRES].

[16] V.D. Barger, M.S. Berger and P. Ohmann, The supersymmetric particle spectrum, Phys. Rev. D 49 (1994) 4908 [hep-ph/9311269] [SPIRES].

[17] D.J. Castano, E.J. Piard and P. Ramond, Renormalization group study of the Standard Model and its extensions. 2. The minimal supersymmetric Standard Model, Phys. Rev. D 49 (1994) 4882 [hep-ph/9308335] [SPIRES]. 
[18] B. Grzadkowski, M. Lindner and S. Theisen, Nonlinear evolution of Yukawa couplings in the double Higgs and supersymmetric extensions of the Standard Model,

Phys. Lett. B 198 (1987) 64 [SPIRES].

[19] S. Antusch, J. Kersten, M. Lindner and M. Ratz, Neutrino mass matrix running for non-degenerate see-saw scales, Phys. Lett. B 538 (2002) 87 [hep-ph/0203233] [SPIRES].

[20] T. Banks, Supersymmetry and the quark mass matrix, Nucl. Phys. B 303 (1988) 172 [SPIRES].

[21] E. Ma, Radiative quark and lepton masses through soft supersymmetry breaking, Phys. Rev. D 39 (1989) 1922 [SPIRES].

[22] R. Hempfling, Yukawa coupling unification with supersymmetric threshold corrections, Phys. Rev. D 49 (1994) 6168 [SPIRES].

[23] L.J. Hall, R. Rattazzi and U. Sarid, The top quark mass in supersymmetric $\mathrm{SO}(10)$ unification, Phys. Rev. D 50 (1994) 7048 [hep-ph/9306309] [SPIRES].

[24] T. Blazek, S. Raby and S. Pokorski, Finite supersymmetric threshold corrections to CKM matrix elements in the large tan $\beta$ regime, Phys. Rev. D 52 (1995) 4151 [hep-ph/9504364] [SPIRES].

[25] M.S. Carena, M. Olechowski, S. Pokorski and C.E.M. Wagner, Electroweak symmetry breaking and bottom-top Yukawa unification, Nucl. Phys. B 426 (1994) 269 [hep-ph/9402253] [SPIRES].

[26] F. Borzumati, G.R. Farrar, N. Polonsky and S.D. Thomas, Soft Yukawa couplings in supersymmetric theories, Nucl. Phys. B 555 (1999) 53 [hep-ph/9902443] [SPIRES].

[27] M.S. Carena, D. Garcia, U. Nierste and C.E.M. Wagner, Effective Lagrangian for the $\bar{t} b H^{+}$ interaction in the MSSM and charged Higgs phenomenology, Nucl. Phys. B 577 (2000) 88 [hep-ph/9912516] [SPIRES].

[28] C. Jarlskog, Commutator of the quark mass matrices in the standard electroweak model and a measure of maximal CP-violation, Phys. Rev. Lett. 55 (1985) 1039 [SPIRES].

[29] M. Battaglia et al., Updated post-WMAP benchmarks for supersymmetry, Eur. Phys. J. C 33 (2004) 273 [hep-ph/0306219] [SPIRES].

[30] B.C. Allanach et al., The Snowmass points and slopes: Benchmarks for SUSY searches, Eur. Phys. J. C 25 (2002) 113 [hep-ph/0202233] [SPIRES].

[31] B. Pontecorvo, Inverse beta processes and nonconservation Hof lepton charge, Sov. Phys. JETP 7 (1958) 172 [Zh. Eksp. Teor. Fiz. 34 (1957) 247] [SPIRES].

[32] Z. Maki, M. Nakagawa and S. Sakata, Remarks on the unified model of elementary particles, Prog. Theor. Phys. 28 (1962) 870 [SPIRES].

[33] A. Dedes and A. Pilaftsis, Resummed effective Lagrangian for Higgs-mediated FCNC interactions in the CP-violating MSSM, Phys. Rev. D 67 (2003) 015012 [hep-ph/0209306] [SPIRES].

[34] S.A. Abel and J.M. Frere, Could the MSSM have no CP-violation in the CKM matrix?, Phys. Rev. D 55 (1997) 1623 [hep-ph/9608251] [SPIRES].

[35] S.R. Coleman and E.J. Weinberg, Radiative corrections as the origin of spontaneous symmetry breaking, Phys. Rev. D 7 (1973) 1888 [SPIRES]. 
[36] L. Hofer, U. Nierste and D. Scherer, Resummation of tan- $\beta$-enhanced supersymmetric loop corrections beyond the decoupling limit, JHEP 10 (2009) 081 [arXiv:0907.5408] [SPIRES].

[37] D.A. Demir, Higgs boson couplings to quarks with supersymmetric CP and flavor violations, Phys. Lett. B 571 (2003) 193 [hep-ph/0303249] [SPIRES].

[38] A. Pilaftsis and C.E.M. Wagner, Higgs bosons in the minimal supersymmetric standard model with explicit CP-violation, Nucl. Phys. B 553 (1999) 3 [hep-ph/9902371] [SPIRES].

[39] D.A. Demir, Effects of the supersymmetric phases on the neutral Higgs sector, Phys. Rev. D 60 (1999) 055006 [hep-ph/9901389] [SPIRES].

[40] S.Y. Choi, M. Drees and J.S. Lee, Loop corrections to the neutral Higgs boson sector of the MSSM with explicit CP-violation, Phys. Lett. B 481 (2000) 57 [hep-ph/0002287] [SPIRES].

[41] M.S. Carena, J.R. Ellis, A. Pilaftsis and C.E.M. Wagner, Renormalization-group-improved effective potential for the MSSM Higgs sector with explicit CP-violation, Nucl. Phys. B 586 (2000) 92 [hep-ph/0003180] [SPIRES].

[42] M.S. Carena, J.R. Ellis, A. Pilaftsis and C.E.M. Wagner, Higgs-boson pole masses in the MSSM with explicit CP-violation, Nucl. Phys. B 625 (2002) 345 [hep-ph/0111245] [SPIRES].

[43] T. Ibrahim and P. Nath, Corrections to the Higgs boson masses and mixings from chargino, $W$ and charged Higgs exchange loops and large CP phases, Phys. Rev. D 63 (2001) 035009 [hep-ph/0008237] [SPIRES].

[44] S.P. Martin, Two-loop effective potential for the minimal supersymmetric standard model, Phys. Rev. D 66 (2002) 096001 [hep-ph/0206136] [SPIRES].

[45] HEIHEI S.F. King and D.A.J. Rayner, Twisted moduli and supersymmetry breaking, JHEP 07 (2002) 047 [hep-ph/0111333] [SPIRES].

[46] J.S. Lee et al., CPsuperH: a computational tool for Higgs phenomenology in the minimal supersymmetric standard model with explicit CP-violation, Comput. Phys. Commun. 156 (2004) 283 [hep-ph/0307377] [SPIRES].

[47] J.S. Lee, M. Carena, J. Ellis, A. Pilaftsis and C.E.M. Wagner, CPsuperH2.0: an improved computational tool for Higgs phenomenology in the MSSM with explicit CP-violation, Comput. Phys. Commun. 180 (2009) 312 [arXiv:0712.2360] [SPIRES].

[48] W. Altmannshofer, A.J. Buras, S. Gori, P. Paradisi and D.M. Straub, Anatomy and phenomenology of FCNC and CPV effects in SUSY theories, arXiv:0909.1333 [SPIRES]. 